\title{
A bird survey in a transitional area between two major conservation hotspots in southeastern Brazil
}

\author{
Guilherme Sementili-Cardoso ${ }^{1,2}$, Renata Marques Vianna ${ }^{1,2}$, Raphael Whitacker Gerotti ${ }^{1,2}$, \\ Reginaldo José Donatelli ${ }^{1}$
}

\begin{abstract}
1 Universidade Estadual Paulista "Júlio de Mesquita Filho" Campus Bauru, Faculdade de Ciências, Departamento de Ciências Biológicas, Laboratório de Ornitologia, Av. Eng. Luiz Edmundo Carrijo Coube, 14-01, Vargem Limpa, Bauru, SP, Brazil, CEP 17033-360, Brazil. 2 Universidade Estadual Paulista "Júlio de Mesquita Filho" Campus Botucatu, Instituto de Biociências, Programa de Pós-graduação em Ciências Biológicas (Zoologia), R. Prof. Dr. Antônio Celso Wagner Zanin, 250, Rubião Junior, Botucatu, SP, CEP 18618-689, Brazil.
\end{abstract}

Corresponding Author: Guilherme Sementili-Cardoso, guisemcar@yahoo.com.br

\begin{abstract}
Transitional areas may have higher species richness than those located within established environments, as these ecotones provide a variety of resources that facilitate the maintenance of specific fauna. However, they tend to be overlooked in wildlife surveys. In this context, the western region of the state of São Paulo in Brazil is important because it contains areas of ecological transition between 2 major conservation hotspots in Brazil: the Atlantic Forest and the Cerrado. We surveyed the avifauna in a transitional area between these domains, using qualitative and quantitative methods between December 2016 and February 2019. We documented 220 species, of which 7 are endemic to the Atlantic Forest and 4 are endemic to the Cerrado. In addition, we documented 9 species that are threatened at some level (regional, national, or global), demonstrating the high conservation value of this area for birds. Therefore, our results show the potential of transitional areas for maintaining regional biodiversity.
\end{abstract}

\section{Keywords}

Avian community, Atlantic Forest, bird assemblage, Cerrado, ecotone.

Academic editor: Rodolpho Rodrigues | Received 3 December 2018 | Accepted 8 April 2019 | Published 28 June 2019

Citation: Guilherme Sementili-Cardoso G, Vianna RM, Gerotti RW, Donatelli RJ (2019) A bird survey in a transitional area between two major conservation hotspots in southeastern Brazil. Check List 15 (3): 527-548. https://doi.org/10.15560/15.3.527

\section{Introduction}

Transitional areas between communities tend to receive less attention in biodiversity research than distinct ecosystems. Studies at the global level tend to assess biodiversity across delimited areas to manage resources efficiently (Myers et al. 2000). However, this approach overlooks transitional areas as valuable biodiversity refuges, despite the relevance of such areas as centers of ecological variability and speciation (Schilthuizen 2000).
Transitional areas (or ecotones) are defined as areas of abrupt transition along an environmental gradient, where abiotic and/or biotic factors change quickly at a small geographic scale (Kark and Van Rensburg 2006). The steep gradient between 2 or among more environments exerts a significant influence on local biodiversity. Such areas tend to have higher species richness and abundance, because they support overlapping communities that would normally be restricted to isolated ecosystems (Kunin 1998, Lloyd et al. 2000, Kucherova and 


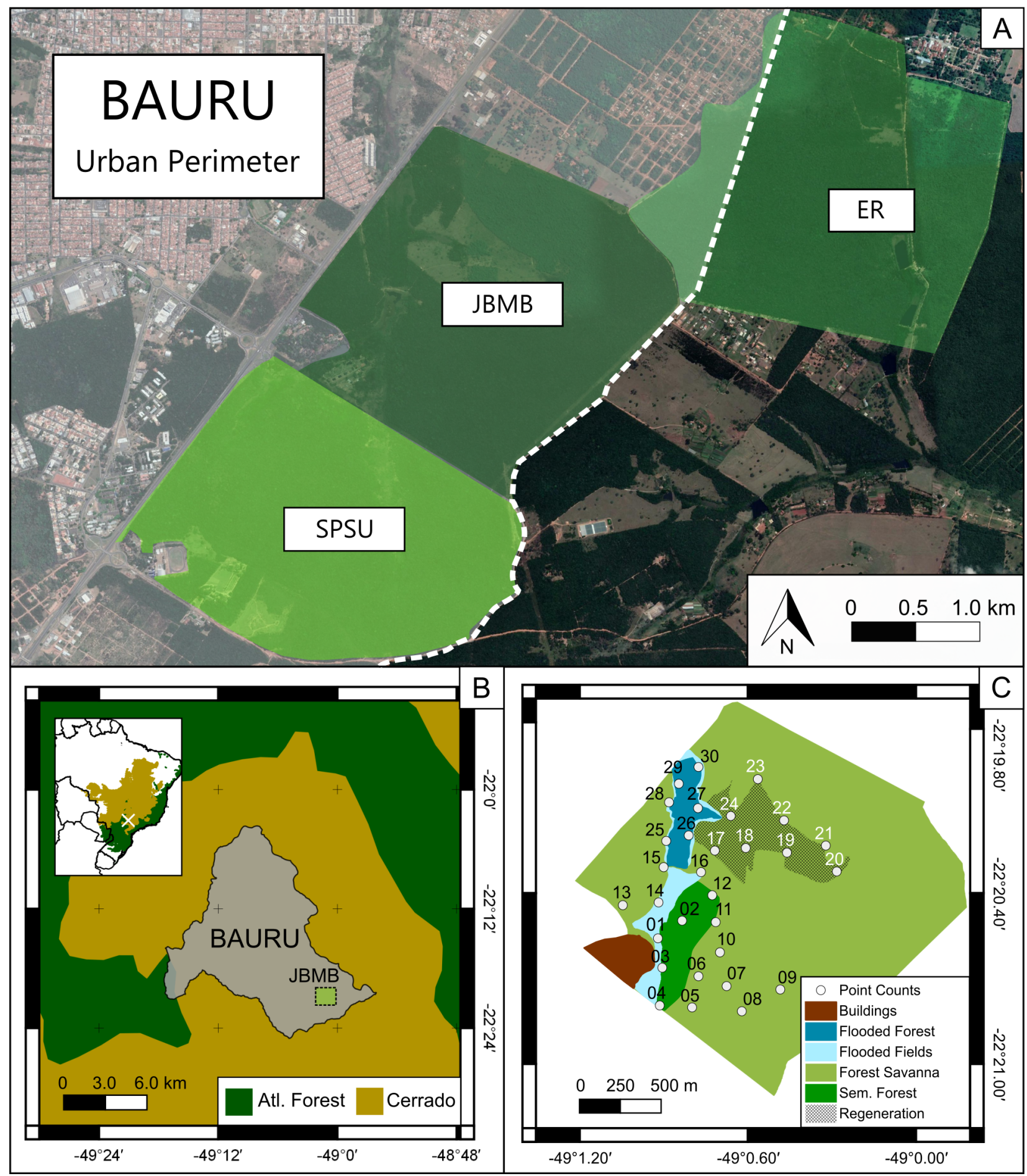

Figure 1. Maps of "Jardim Botânico Municipal de Bauru" (JBMB) region. A. Satellite image of the region, displaying the urban perimeter of Bauru (white shaded area) and the two JBMB's adjacent fragments (SPSU = São Paulo State University legal reserve, ER = Ecological Reserve of "Sociedade Beneficente Enéas Carvalho de Aguiar"). B. Area of Bauru. The city is inserted in a transitional region between Atlantic Forest (green) and Cerrado (yellow). C. Area of JBMB, including the locations of point counts (white dots), and the predominant physiognomies found in JBMB. Each number corresponds to a different point count. White numbers refer to the points located inside the regenerating area

Mirkin 2001, Baker et al. 2002, Dangerfield et al. 2003, Kark et al. 2007). In addition, transitional areas may contain unique richness (Odum 1953) because the combined diversity of adjacent sites facilitates the maintenance of rare morphological, genetic, and ecological features of biological taxa (Kark and van Rensburg 2006). In addition, some authors hypothesized that transitional areas are zones where new species could emerge more quickly (Moritz et al. 2000), especially in bird taxa (Kark et al. 2007).

In this context, the southeastern region of Brazil plays an important role in the maintenance of bird diversity because it contains several ecotones of Cerrado and Atlantic Forest ecoregions (São Paulo 2017). Both 


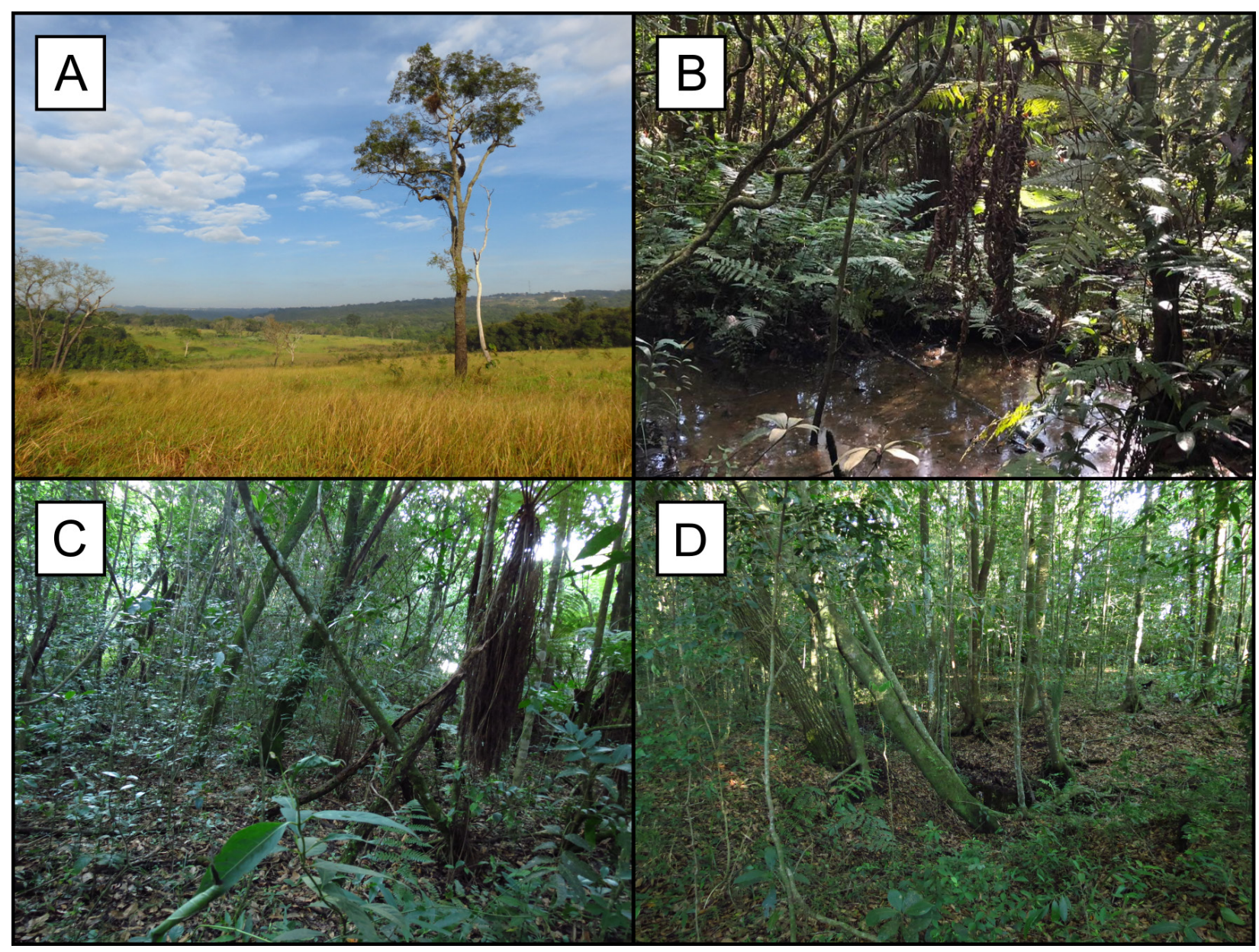

Figure 2. Vegetal physiognomy and vegetal structure variation in JBMB. A. regenerating area. B. Semideciduous Flooded Forest. C. Semideciduous Forest. D. Forest Savanna ("Cerradão").

ecoregions support high biodiversity and high degree of endemisms, with several species being threatened by the high rates of habitat loss in them (Myers et al. 2000). Consequently, this region could be fundamental for the conservation of both ecoregions biodiversity. Information on bird distribution and community structure in such environments is crucial to conservation and management (Kark and van Rensburg 2006) and fauna inventories are critical for understanding the composition and dynamics of such communities (Santos 2003). Therefore, our work presents a detailed inventory of avian taxa in an important ecotone of southeastern Brazil and emphasizes the occurrence of those species that present high conservation value, such as endemic, threatened, or migratory species.

\section{Methods}

The Jardim Botânico Municipal de Bauru (hereafter JBMB) is located at Bauru Municipality $\left(22^{\circ} 19^{\prime} \mathrm{S}\right.$, $049^{\circ} 04^{\prime} \mathrm{W}$ ) in the midwestern region of São Paulo state (Fig. 1). According to Alvares et al. (2013), this region climate is defined as Cwa by Köppen classification, with 2 well-defined seasons of hot and wet summers versus cold and dry winters. JBMB covers a total area of 321 ha and is one of three conservation units that integrates the Vargem Limpa-Campo Novo Area of Environmental Protection (Bauru 2000), along with the São Paulo State University Legal Reserve (265 ha) and the Ecological Reserve of "Sociedade Beneficente Enéas Carvalho de Aguiar" (217 ha). Together, these three conservation units encompasses more than 800 ha of native vegetation (Cavassan 2013).

There are different vegetal formations (or phytophysiognomies) in JBMB that belong to the Atlantic Forest and Cerrado domains (Cavassan 2013). For instance, Forest Savanna (which is known locally as Cerradão) occupies 280 ha; Semideciduous Forest (Veloso 1992) occupies 7 ha; and Riparian Semideciduous Forest with permanent fluvial influence (which is known as Semidecidual Flooded Forest) occupies 11 ha (Rodrigues 2000). In addition, the JBMB contains about 15 ha Regenerating Forest Savanna (Weiser 2007) (Fig. 2). All these phytophysiognomies are distributed in an area of more than 300 ha, which makes this remnant one of the most important fragments of high priority for conservation in the midwestern state of São Paulo (Kronka et al. 1998, Durigan et al. 2004).

To document avifauna in the JBMB, we used 10-min point counts, which had no sampling distance limit (Blondel et al. 1981) and were adapted to tropical regions (Vielliard and Silva 1990). We sampled 30 points 
separated by a minimum distance of $200 \mathrm{~m}$ from each other, distributing them between the phytophysiognomies of JBMB (Table 1). We surveyed the points twice a month, in the mornings (06:00-10:00) and in the afternoons (15:00-19:00). Sampling was conducted monthly in 2 stages. Stage 1 was conducted from December 2016 to December 2017 in the Forest Savanna, Semideciduous Forest, and Regenerating area. Stage 2 was conducted from February 2018 to February 2019 in the Riparian Forest. In total, we completed $120 \mathrm{~h}$ of avian surveys.

Visual records were made using Nikon PROSTAFF 7S $10 \times 42$ binoculars, while sound records were made with a Marantz PMD-667 recorder coupled to a Yoga HT-81 directional microphone. Photographic records of the species were obtained using a Canon Powershot Superzoom $40 \times$ digital camera whenever possible. Pairs of researchers (GSC, RMV, RWG) carried out the observations to avoid observation bias. To complement the bird survey, we recorded sporadic contacts occurring outside sampling period, both within the JBMB and adjacent areas. We did not record the abundance of species, because this survey was descriptive in nature. In addition, we integrated the internal database of JBMB species to our list to complement our own recordings.

Patterns of migratory activity were classified according to Somenzari et al. (2018), with some changes. We considered migratory species (MGT) as those that leave their territory after the reproductive season and return to the same area after a cyclical period. Partially migratory species (MPR) included populations with some migratory individuals, while others remained resident in certain regions. The endemism status of each species was based on Bencke et al. (2006) for the Atlantic Forest and on Silva and Bates (2002) for the Cerrado. The Red List category of each species was defined at a global level based on the International Union for Conservation of Nature (IUCN 2019), at a national level based on Livro Vermelho da Fauna Brasileira Ameaçada de Extinção (ICMBIO/MMA 2018), and at a regional level based on the State Decree No. 63.853 (São Paulo 2018). We also identified species subjected to capture and traffic pressure according to Costa and Monteiro (2016). Taxonomic ordering and nomenclature followed the recommendations of the Brazilian Ornithological Records Committee (Piacentini et al. 2015).

\section{Results}

Across all surveys and data sources, we recorded 220 species (Table 2) belonging to 22 orders and 54 families. Thirty-six species (16.4\% of the total) were documented by photographs (Figs 3-8). Of the 54 families, 23 belonged to the order Passeriformes and 31 belonged

Table 1. List of point counts used in the avifaunal survey of the Jardim Botânico Municipal de Bauru (JBMB), with the sampling period, the geographic coordinates and the predominant vegetation of each point.

\begin{tabular}{|c|c|c|c|c|}
\hline Point no. & Sampling period & Latitude & Longitude & Vegetation \\
\hline 01 & Dec. 2016-Dec. 2017 & $22^{\circ} 20^{\prime} 35^{\prime \prime} \mathrm{S}$ & $49^{\circ} 00^{\prime} 57^{\prime \prime} \mathrm{W}$ & Semideciduous Forest \\
\hline 02 & Dec. 2016-Dec. 2017 & $22^{\circ} 20^{\prime} 35^{\prime \prime} \mathrm{S}$ & $49^{\circ} 00^{\prime} 50^{\prime \prime} \mathrm{W}$ & Semideciduous Forest \\
\hline 03 & Dec. 2016-Dec. 2017 & $22^{\circ} 20^{\prime} 40^{\prime \prime} \mathrm{S}$ & $49^{\circ} 00^{\prime} 55^{\prime \prime} \mathrm{W}$ & Semideciduous Forest \\
\hline 04 & Dec. 2016-Dec. 2017 & $22^{\circ} 20^{\prime} 48^{\prime \prime} \mathrm{S}$ & $49^{\circ} 00^{\prime} 55^{\prime \prime} \mathrm{W}$ & Semideciduous Forest \\
\hline 05 & Dec. 2016-Dec. 2017 & $22^{\circ} 20^{\prime} 48^{\prime \prime} \mathrm{S}$ & $49^{\circ} 00^{\prime} 48^{\prime \prime} \mathrm{W}$ & Forest Savanna \\
\hline 06 & Dec. 2016-Dec. 2017 & $22^{\circ} 20^{\prime} 41^{\prime \prime} \mathrm{S}$ & $49^{\circ} 00^{\prime} 46^{\prime \prime} \mathrm{W}$ & Forest Savanna \\
\hline 07 & Dec. 2016-Dec. 2017 & $22^{\circ} 20^{\prime} 43^{\prime \prime} \mathrm{S}$ & $49^{\circ} 00^{\prime} 40^{\prime \prime} \mathrm{W}$ & Forest Savanna \\
\hline 08 & Dec. 2016-Dec. 2017 & $22^{\circ} 20^{\prime} 48^{\prime \prime} \mathrm{S}$ & $49^{\circ} 00^{\prime} 37^{\prime \prime} \mathrm{W}$ & Forest Savanna \\
\hline 09 & Dec. 2016-Dec. 2017 & $22^{\circ} 20^{\prime} 44^{\prime \prime} \mathrm{S}$ & $49^{\circ} 00^{\prime} 29^{\prime \prime} \mathrm{W}$ & Forest Savanna \\
\hline 10 & Dec. 2016-Dec. 2017 & $22^{\circ} 20^{\prime} 36^{\prime \prime} \mathrm{S}$ & $49^{\circ} 00^{\prime} 42^{\prime \prime} \mathrm{W}$ & Forest Savanna \\
\hline 11 & Dec. 2016-Dec. 2017 & $22^{\circ} 20^{\prime} 31^{\prime \prime} \mathrm{S}$ & $49^{\circ} 00^{\prime} 41^{\prime \prime} \mathrm{W}$ & Semideciduous Forest \\
\hline 12 & Dec. 2016-Dec. 2017 & $22^{\circ} 20^{\prime} 28^{\prime \prime} \mathrm{S}$ & $49^{\circ} 00^{\prime} 47^{\prime \prime} \mathrm{W}$ & Semideciduous Forest \\
\hline 13 & Dec. 2016-Dec. 2017 & $22^{\circ} 20^{\prime} 28^{\prime \prime} \mathrm{S}$ & $49^{\circ} 01^{\prime} 02^{\prime \prime} \mathrm{W}$ & Forest Savanna \\
\hline 14 & Dec. 2016-Dec. 2017 & $22^{\circ} 20^{\prime} 26^{\prime \prime} \mathrm{S}$ & $49^{\circ} 00^{\prime} 55^{\prime \prime} \mathrm{W}$ & Flooded Field \\
\hline 15 & Dec. 2016-Dec. 2017 & $22^{\circ} 20^{\prime} 18^{\prime \prime} \mathrm{S}$ & $49^{\circ} 00^{\prime} 54^{\prime \prime} \mathrm{W}$ & Flooded Field \\
\hline 16 & Dec. 2016-Dec. 2017 & $22^{\circ} 20^{\prime} 21^{\prime \prime} \mathrm{S}$ & $49^{\circ} 00^{\prime} 46^{\prime \prime} \mathrm{W}$ & Flooded Field \\
\hline 17 & Dec. 2016-Dec. 2017 & $22^{\circ} 20^{\prime} 15^{\prime \prime} \mathrm{S}$ & $49^{\circ} 00^{\prime} 44^{\prime \prime} \mathrm{W}$ & Regenerating Area \\
\hline 18 & Dec. 2016-Dec. 2017 & $22^{\circ} 20^{\prime} 14^{\prime \prime} \mathrm{S}$ & $49^{\circ} 00^{\prime} 36^{\prime \prime} \mathrm{W}$ & Regenerating Area \\
\hline 19 & Dec. 2016-Dec. 2017 & $22^{\circ} 20^{\prime} 15^{\prime \prime} \mathrm{S}$ & $49^{\circ} 00^{\prime} 27^{\prime \prime} \mathrm{W}$ & Regenerating Area \\
\hline 20 & Dec. 2016-Dec. 2017 & $22^{\circ} 20^{\prime} 19^{\prime \prime} \mathrm{S}$ & $49^{\circ} 00^{\prime} 17^{\prime \prime} \mathrm{W}$ & Regenerating Area \\
\hline 21 & Dec. 2016-Dec. 2017 & $22^{\circ} 20^{\prime} 14^{\prime \prime} \mathrm{S}$ & $49^{\circ} 00^{\prime} 19^{\prime \prime} \mathrm{W}$ & Regenerating Area \\
\hline 22 & Dec. 2016-Dec. 2017 & $22^{\circ} 20^{\prime} 08^{\prime \prime} \mathrm{S}$ & $49^{\circ} 00^{\prime} 28^{\prime \prime} \mathrm{W}$ & Regenerating Area \\
\hline 23 & Dec. 2016-Dec. 2017 & $22^{\circ} 19^{\prime} 60^{\prime \prime} \mathrm{S}$ & $49^{\circ} 00^{\prime} 34^{\prime \prime} \mathrm{W}$ & Regenerating Area \\
\hline 24 & Dec. 2016-Dec. 2017 & $22^{\circ} 20^{\prime} 07^{\prime \prime} \mathrm{S}$ & $49^{\circ} 00^{\prime} 39^{\prime \prime} \mathrm{W}$ & Regenerating Area \\
\hline 25 & Feb. 2018-Feb. 2019 & $22^{\circ} 20^{\prime} 12^{\prime \prime} \mathrm{S}$ & $49^{\circ} 00^{\prime} 53^{\prime \prime} \mathrm{W}$ & Riparian Forest \\
\hline 26 & Feb. 2018-Feb. 2019 & $22^{\circ} 20^{\prime} 12^{\prime \prime} \mathrm{S}$ & $49^{\circ} 00^{\prime} 48^{\prime \prime} \mathrm{W}$ & Riparian Forest \\
\hline 27 & Feb. 2018-Feb. 2019 & $22^{\circ} 20^{\prime} 08^{\prime \prime} \mathrm{S}$ & $49^{\circ} 00^{\prime} 48^{\prime \prime} \mathrm{W}$ & Riparian Forest \\
\hline 28 & Feb. 2018-Feb. 2019 & $22^{\circ} 20^{\prime} 05^{\prime \prime} \mathrm{S}$ & $49^{\circ} 00^{\prime} 53^{\prime \prime} \mathrm{W}$ & Riparian Forest \\
\hline 29 & Feb. 2018-Feb. 2019 & $22^{\circ} 20^{\prime} 01^{\prime \prime} \mathrm{S}$ & $49^{\circ} 00^{\prime} 50^{\prime \prime} \mathrm{W}$ & Riparian Forest \\
\hline 30 & Feb. 2018-Feb. 2019 & $22^{\circ} 19^{\prime} 57^{\prime \prime} \mathrm{S}$ & $49^{\circ} 00^{\prime} 46^{\prime \prime} \mathrm{W}$ & Riparian Forest \\
\hline
\end{tabular}




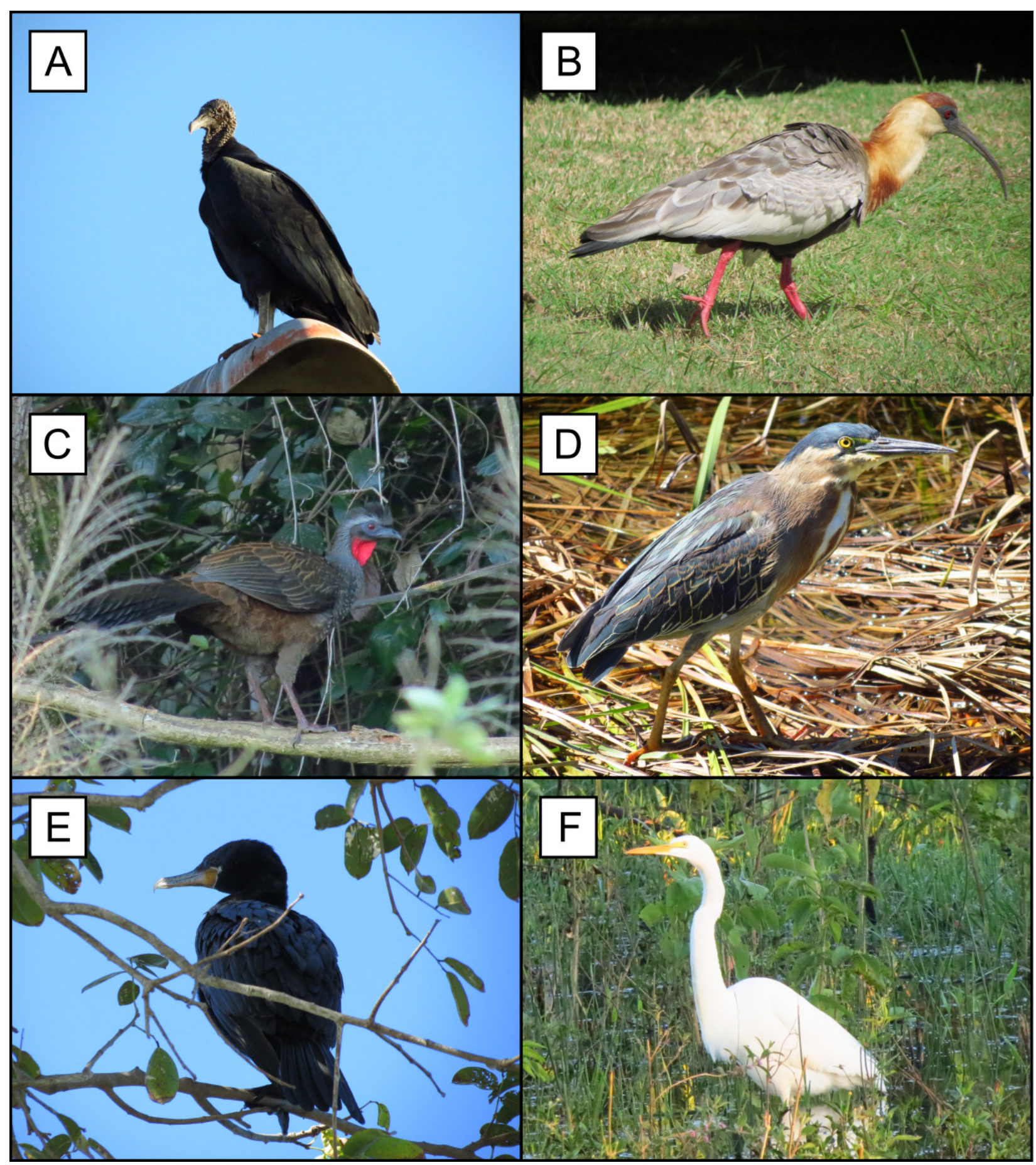

Figure 3. Birds recorded during our survey at the JBMB. A. Black Vulture Coragyps atratus (point no. 12). B. Buff-necked Ibis Theristicus caudatus (point no. 01). C. Rusty-margined Guan Penelope superciliaris (point no. 03). D. Striated Heron Butorides striata (point no. 3). E. Neotropic Cormorant Nannopterum brasilianus (point no. 14) F. Great Egret Ardea alba (point no. 14).

to other orders. We observed 190 species $(86.3 \%$ of the total) in quantitative surveys and 26 species $(11.8 \%$ of the total) in qualitative surveys. Just 4 species $(1.9 \%$ of the total) were only found in the JBMB database. Among non-Passeriformes families, the Accipitridae, Columbidae, and Trochilidae had the highest richness ( 9 species each, $4.1 \%$ of the total), followed by Ardeidae and Rallidae ( 7 species each, $3.2 \%$ of the total). Among the Passeriformes, 30 species represented the Tyrannidae $(13.7 \%$ of the total) and 27 species represented the Thraupidae (12.4\% of the total) families. We recorded 31 species
(14.2\% of the total) with migratory activity, with 2 species being considered migratory and 29 species being considered partially migratory,. In addition, we documented 36 species ( $16.5 \%$ of the total) subjected to capture and traffic pressure (Costa and Monteiro 2016). We also documented 2 exotic species, Estrilda astrild (Common Waxbill) and Passer domesticus (House Sparrow).

Some species that were documented are considered of conservation relevance. Among the species classified as endemic, we recorded 7 taxa restricted to the Atlantic Forest and 4 restricted to the Cerrado. 
Table 2. List of birds recorded at Jardim Botânico Municipal de Bauru (JBMB), with the scientific name and English names. We also display the method of detection (Quan. = Quantitative survey, Qual. = Qualitative survey, Data. = JBMB databases); the predominant physiognomy (Phys.) where the taxa have been found (SEM = Semideciduous Forest, REG = Regenerating area, SAV = Forest Savanna, WET = Wetlands, $\mathrm{UBQ}=$ Ubiquitous); the migratory behavior (Mig.) of each taxa (MGT = migratory species, MPR = partially migratory species); the conservation status at the global (IUCN 2019), national (ICMBIO/MMA 2018) and regional (São Paulo 2018) levels, respectively (LC = least concern, NT = near threatened, $\mathrm{VU}=$ vulnerable, $\mathrm{EN}=$ endangered); and the taxa that suffers from risk of capture and traffic pressures (Traf.) according to Costa and Monteiro (2016).

\begin{tabular}{|c|c|c|c|c|c|c|c|c|}
\hline Taxon & English name & Quan. & Qual. & Data. & Phys. & Mig. & $\begin{array}{c}\text { Conserv. } \\
\text { status }\end{array}$ & Traf. \\
\hline \multicolumn{9}{|l|}{ TINAMIFORMES } \\
\hline \multicolumn{9}{|l|}{ Tinamidae } \\
\hline Crypturellus obsoletus (Temminck, 1815) & Brown Tinamou & $x$ & & & SEM & & $\mathrm{LC} / \mathrm{LC} / \mathrm{LC}$ & \\
\hline Crypturellus parvirostris (Wagler, 1827) & Small-billed Tinamou & $\mathrm{x}$ & & & REG & & $\mathrm{LC} / \mathrm{LC} / \mathrm{LC}$ & \\
\hline Crypturellus tataupa (Temminck, 1815) & Tataupa Tinamou & $\mathrm{x}$ & & & SEM/REG & & $\mathrm{LC} / \mathrm{LC} / \mathrm{LC}$ & \\
\hline Rhynchotus rufescens (Temminck, 1815) & Red-winged Tinamou & & $x$ & & REG & & $\mathrm{LC} / \mathrm{LC} / \mathrm{EN}$ & \\
\hline \multicolumn{9}{|l|}{ ANSERIFORMES } \\
\hline \multicolumn{9}{|l|}{ Anatidae } \\
\hline Cairina moschata (Linnaeus, 1758) & Muscovy Duck & $x$ & & & WET & & $\mathrm{LC} / \mathrm{LC} / \mathrm{LC}$ & \\
\hline Amazonetta brasiliensis (Gmelin, 1789) & Brazilian Teal & & $x$ & & WET & & $\mathrm{LC} / \mathrm{LC} / \mathrm{LC}$ & \\
\hline \multicolumn{9}{|l|}{ GALLIFORMES } \\
\hline \multicolumn{9}{|l|}{ Cracidae } \\
\hline Penelope superciliaris Temminck, 1815 & Rusty-margined Guan & $x$ & & & SEM & & $\mathrm{LC} / \mathrm{LC} / \mathrm{NT}$ & \\
\hline \multicolumn{9}{|l|}{ PODICIPEDIFORMES } \\
\hline \multicolumn{9}{|l|}{ Podicipedidae } \\
\hline Podilymbus podiceps (Linnaeus, 1758) & Pied-billed Grebe & & $x$ & & WET & & $\mathrm{LC} / \mathrm{LC} / \mathrm{LC}$ & \\
\hline \multicolumn{9}{|l|}{ SULIFORMES } \\
\hline \multicolumn{9}{|l|}{ Phalacrocoracidae } \\
\hline Nannopterum brasilianus (Gmelin, 1789) & Neotropic Cormorant & & $x$ & & WET & & $\mathrm{LC} / \mathrm{LC} / \mathrm{LC}$ & \\
\hline \multicolumn{9}{|l|}{ Anhingidae } \\
\hline Anhinga anhinga (Linnaeus, 1766) & Anhinga & $x$ & & & WET & & $\mathrm{LC} / \mathrm{LC} / \mathrm{LC}$ & \\
\hline \multicolumn{9}{|l|}{ Ardeidae } \\
\hline Tigrisoma lineatum (Boddaert, 1783) & Rufescent Tiger-Heron & $x$ & & & WET & & $\mathrm{LC} / \mathrm{LC} / \mathrm{LC}$ & \\
\hline Butorides striata (Linnaeus, 1758) & Striated Heron & & $x$ & & WET & & $\mathrm{LC} / \mathrm{LC} / \mathrm{LC}$ & \\
\hline Bubulcus ibis (Linnaeus, 1758) & Cattle Egret & & $x$ & & WET & & $\mathrm{LC} / \mathrm{LC} / \mathrm{LC}$ & \\
\hline Ardea cocoi Linnaeus, 1766 & Cocoi Heron & & $x$ & & WET & & $\mathrm{LC} / \mathrm{LC} / \mathrm{LC}$ & \\
\hline Ardea alba Linnaeus, 1758 & Great Egret & $x$ & & & WET & & $\mathrm{LC} / \mathrm{LC} / \mathrm{LC}$ & \\
\hline Syrigma sibilatrix (Temminck, 1824) & Whistling Heron & $x$ & & & WET & & $\mathrm{LC} / \mathrm{LC} / \mathrm{LC}$ & \\
\hline Egretta thula (Molina, 1782) & Snowy Egret & $x$ & & & WET & & $\mathrm{LC} / \mathrm{LC} / \mathrm{LC}$ & \\
\hline \multicolumn{9}{|l|}{ Threskiornithidae } \\
\hline Mesembrinibis cayennensis (Gmelin, 1789) & Green Ibis & $x$ & & & WET & & $\mathrm{LC} / \mathrm{LC} / \mathrm{LC}$ & \\
\hline Phimosus infuscatus (Lichtenstein, 1823) & Bare-faced lbis & $x$ & & & WET & & $\mathrm{LC} / \mathrm{LC} / \mathrm{LC}$ & \\
\hline Theristicus caudatus (Boddaert, 1783) & Buff-necked lbis & $x$ & & & UBQ & & $\mathrm{LC} / \mathrm{LC} / \mathrm{LC}$ & \\
\hline \multicolumn{9}{|l|}{ CATHARTIFORMES } \\
\hline \multicolumn{9}{|l|}{ Cathartidae } \\
\hline Cathartes aura (Linnaeus, 1758) & Turkey Vulture & $x$ & & & REG & & $\mathrm{LC} / \mathrm{LC} / \mathrm{LC}$ & \\
\hline Coragyps atratus (Bechstein, 1793) & Black Vulture & $x$ & & & UBQ & & $\mathrm{LC} / \mathrm{LC} / \mathrm{LC}$ & \\
\hline \multicolumn{9}{|l|}{ ACCIPITRIFORMES } \\
\hline \multicolumn{9}{|l|}{ Accipitridae } \\
\hline Chondrohierax uncinatus (Temminck, 1822) & Hook-billed Kite & & $x$ & & WET & & $\mathrm{LC} / \mathrm{LC} / \mathrm{LC}$ & \\
\hline Gampsonyx swainsonii Vigors, 1825 & Pearl Kite & $x$ & & & SAV/SEM & & $\mathrm{LC} / \mathrm{LC} / \mathrm{LC}$ & \\
\hline Elanus leucurus (Vieillot, 1818) & White-tailed Kite & $x$ & & & SAV/SEM & & $\mathrm{LC} / \mathrm{LC} / \mathrm{LC}$ & \\
\hline Ictinia plumbea (Gmelin, 1788) & Plumbeous Kite & $x$ & & & SAV/SEM & MPR & $\mathrm{LC} / \mathrm{LC} / \mathrm{LC}$ & \\
\hline Rostrhamus sociabilis (Vieillot, 1817) & Snail Kite & & $x$ & & WET & MPR & $\mathrm{LC} / \mathrm{LC} / \mathrm{LC}$ & \\
\hline Heterospizias meridionalis (Latham, 1790) & Savanna Hawk & & $x$ & & REG & & $\mathrm{LC} / \mathrm{LC} / \mathrm{LC}$ & \\
\hline Rupornis magnirostris (Gmelin, 1788) & Roadside Hawk & $x$ & & & UBQ & & $\mathrm{LC} / \mathrm{LC} / \mathrm{LC}$ & \\
\hline Geranoaetus albicaudatus (Vieillot, 1816) & White-tailed Hawk & & $x$ & & REG & & $\mathrm{LC} / \mathrm{LC} / \mathrm{LC}$ & \\
\hline Buteo brachyurus Vieillot, 1816 & Short-tailed Hawk & $x$ & & & SAV/SEM & & $\mathrm{LC} / \mathrm{LC} / \mathrm{LC}$ & \\
\hline GRUIFORMES & & & & & & & & \\
\hline Aramidae & & & & & & & & \\
\hline Aramus guarauna (Linnaeus, 1766) & Limpkin & $x$ & & & WET & & $\mathrm{LC} / \mathrm{LC} / \mathrm{LC}$ & \\
\hline Rallidae & & & & & & & & \\
\hline Aramides cajaneus (Statius Müller, 1776) & Gray-necked Wood-Rail & $x$ & & & WET & & $\mathrm{LC} / \mathrm{LC} / \mathrm{LC}$ & \\
\hline Laterallus viridis (Statius Müller, 1776) & Russet-crowned Crake & $x$ & & & WET & & $\mathrm{LC} / \mathrm{LC} / \mathrm{LC}$ & \\
\hline Laterallus melanophaius (Vieillot, 1819) & Rufous-sided Crake & $\mathrm{x}$ & & & WET & & $\mathrm{LC} / \mathrm{LC} / \mathrm{LC}$ & \\
\hline
\end{tabular}




\begin{tabular}{|c|c|c|c|c|c|c|c|c|}
\hline Taxon & English name & Quan. & Qual. & Data. & Phys. & Mig. & $\begin{array}{c}\text { Conserv. } \\
\text { status }\end{array}$ & Traf. \\
\hline Mustelirallus albicollis (Vieillot, 1819) & Ash-throated Crake & $\mathrm{x}$ & & & WET & & $\mathrm{LC} / \mathrm{LC} / \mathrm{LC}$ & \\
\hline Pardirallus nigricans (Vieillot, 1819) & Blackish Rail & $x$ & & & WET & & $\mathrm{LC} / \mathrm{LC} / \mathrm{LC}$ & \\
\hline Gallinula galeata (Lichtenstein, 1818) & Common Gallinule & $x$ & & & WET & & $\mathrm{LC} / \mathrm{LC} / \mathrm{LC}$ & \\
\hline Porphyrio martinicus (Linnaeus, 1766) & Purple Gallinule & & $x$ & & WET & MPR & LC/ LC/ LC & \\
\hline \multicolumn{9}{|l|}{ Heliornithidae } \\
\hline Heliornis fulica (Boddaert, 1783) & Sungrebe & & $\mathrm{x}$ & & WET & & LC/ LC/ EN & \\
\hline
\end{tabular}

\section{Charadriidae}

Vanellus chilensis (Molina, 1782)

Southern Lapwing

$X$

REG

LC/ LC/ LC

Jacanidae

Jacana jacana (Linnaeus, 1766)

Wattled Jacana

$\mathrm{X}$

WET

LC/ LC/ LC

\section{COLUMBIFORMES}

Columbidae

Columbina talpacoti (Temminck, 1810)

Columbina squammata (Lesson, 1831)

Claravis pretiosa (Ferrari-Perez, 1886)

Patagioenas picazuro (Temminck, 1813)

Patagioenas cayennensis (Bonnaterre, 1792)

Zenaida auriculata (Des Murs, 1847)

Leptotila verreauxi Bonaparte, 1855

Leptotila rufaxilla (Richard \& Bernard, 1792)

Geotrygon montana (Linnaeus, 1758)

\section{CUCULIFORMES}

\section{Cuculidae}

Piaya cayana (Linnaeus, 1766)

Crotophaga major Gmelin, 1788

Crotophaga ani Linnaeus, 1758

Guira guira (Gmelin, 1788)

Tapera naevia (Linnaeus, 1766)

\section{STRIGIFORMES}

Tytonidae

Tyto furcata (Temminck, 1827)

Strigidae

Megascops choliba (Vieillot, 1817)

Bubo virginianus (Gmelin, 1788)

Athene cunicularia (Molina, 1782)

Asio stygius (Wagler, 1832)

NYCTIBIIFORMES

Nyctibiidae

Nyctibius griseus (Gmelin, 1789)

\section{CAPRIMULGIFORMES}

Caprimulgidae

Lurocalis semitorquatus (Gmelin, 1789)

Nyctidromus albicollis (Gmelin, 1789)

Hydropsalis parvula (Gould, 1837)

Chordeiles minor (Forster, 1771)

\section{APODIFORMES}

Apodidae

Chaetura meridionalis Hellmayr, 1907

Trochilidae

Phaethornis pretrei (Lesson \& Delattre, 1839)

Eupetomena macroura (Gmelin, 1788)

Florisuga fusca (Vieillot, 1817)

Colibri serrirostris (Vieillot, 1816)

Chlorostilbon lucidus (Shaw, 1812)

Thalurania glaucopis (Gmelin, 1788) ATL

Hylocharis chrysura (Shaw, 1812)

Amazilia lactea (Lesson, 1832)

Heliomaster squamosus (Temminck, 1823)

\section{CORACIIFORMES}

Alcedinidae

Megaceryle torquata (Linnaeus, 1766)

$\begin{array}{lccc}\text { Ruddy Ground-Dove } & X & \text { REG } & \mathrm{LC} / \mathrm{LC} / \mathrm{LC} \\ \text { Scaled Dove } & X & \text { REG } & \mathrm{LC} / \mathrm{LC} / \mathrm{LC} \\ \text { Blue Ground-Dove } & X & \mathrm{SEM} & \mathrm{LC} / \mathrm{LC} / \mathrm{LC} \\ \text { Picazuro Pigeon } & \mathrm{X} & \mathrm{UBQ} & \mathrm{LC} / \mathrm{LC} / \mathrm{LC} \\ \text { Pale-vented Pigeon } & X & \mathrm{SAV} / \mathrm{REG} & \mathrm{LC} / \mathrm{LC} / \mathrm{LC} \\ \text { Eared Dove } & X & \mathrm{UBQ} & \mathrm{LC} / \mathrm{LC} / \mathrm{LC} \\ \text { White-tipped Dove } & X & \mathrm{SAV} / \mathrm{REG} & \mathrm{LC} / \mathrm{LC} / \mathrm{LC} \\ \text { Gray-fronted Dove } & X & \text { SAV/REG } & \mathrm{LC} / \mathrm{LC} / \mathrm{LC} \\ \text { Ruddy Quail-Dove } & X & \text { SEM } & \mathrm{LC} / \mathrm{LC} / \mathrm{LC}\end{array}$

Ruddy Quail-Dove

$\begin{array}{llll}\text { Squirrel Cuckoo } & X & \text { UBQ } & \text { LC/ LC/ LC } \\ \text { Greater Ani } & & \text { WET } & \text { LC/ LC/ NT } \\ \text { Smooth-billed Ani } & X & \text { REG } & \text { LC/ LC/ LC } \\ \text { Guira Cuckoo } & X & \text { REG } & \text { LC/ LC/ LC } \\ \text { Striped Cuckoo } & X & \text { REG } & \text { LC/ LC/ LC }\end{array}$

\begin{tabular}{|c|c|c|c|c|}
\hline American Barn Owl & & $x$ & SAV/REG & $\mathrm{LC} / \mathrm{LC} / \mathrm{LC}$ \\
\hline Tropical Screech-Owl & $x$ & & SAV/REG & $\mathrm{LC} / \mathrm{LC} / \mathrm{LC}$ \\
\hline Great Horned Owl & $\mathrm{x}$ & & SAV/REG & $\mathrm{LC} / \mathrm{LC} / \mathrm{EN}$ \\
\hline Burrowing Owl & $\mathrm{x}$ & & REG & $\mathrm{LC} / \mathrm{LC} / \mathrm{LC}$ \\
\hline Stygian Owl & & $x$ & SAV & $\mathrm{LC} / \mathrm{LC} / \mathrm{LC}$ \\
\hline
\end{tabular}

Common Potoo

X

SAV

$\mathrm{LC} / \mathrm{LC} / \mathrm{LC}$

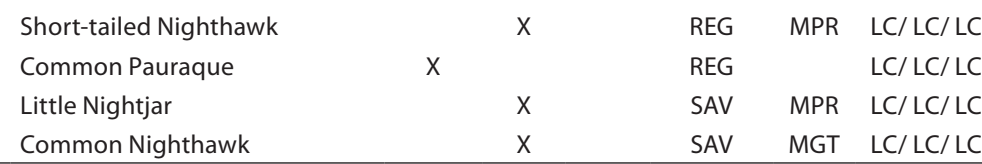

Common Nighthawk

MGT LC/ LC/ LC

\begin{tabular}{llccc} 
Sick's Swift & $X$ & REG & MGT & LC/ LC/ LC \\
Planalto Hermit & $X$ & & UBQ & LC/ LC/ LC \\
Swallow-tailed Hummingbird & $X$ & & UBQ & $\mathrm{LC} / \mathrm{LC} / \mathrm{LC}$ \\
Black Jacobin & $\mathrm{X}$ & $\mathrm{UBQ}$ & $\mathrm{MPR}$ & $\mathrm{LC} / \mathrm{LC} / \mathrm{LC}$ \\
White-vented Violetear & $\mathrm{X}$ & $\mathrm{SEM}$ & $\mathrm{LC} / \mathrm{LC} / \mathrm{LC}$ \\
Glittering-bellied Emerald & $\mathrm{X}$ & & $\mathrm{UBQ}$ & $\mathrm{LC} / \mathrm{LC} / \mathrm{LC}$ \\
Violet-capped Woodnymph & & $\mathrm{X}$ & $\mathrm{SEM}$ & $\mathrm{LC} / \mathrm{LC} / \mathrm{LC}$ \\
Gilded Hummingbird & & $\mathrm{X}$ & $\mathrm{SEM}$ & $\mathrm{LC} / \mathrm{LC} / \mathrm{LC}$ \\
Sapphire-spangled Emerald & $\mathrm{X}$ & & $\mathrm{UBQ}$ & $\mathrm{LC} / \mathrm{LC} / \mathrm{LC}$ \\
Stripe-breasted Starthroat & & & $\mathrm{REG}$ & $\mathrm{LC} / \mathrm{LC} / \mathrm{LC}$ \\
\hline
\end{tabular}

Stripe-breasted Starthroat

REG

LC/ LC/ LC 


\begin{tabular}{|c|c|c|c|c|c|c|c|c|}
\hline Taxon & English name & Quan. & Qual. & Data. & Phys. & Mig. & $\begin{array}{c}\text { Conserv. } \\
\text { status }\end{array}$ & Traf. \\
\hline Chloroceryle amazona (Latham, 1790) & Amazon Kingfisher & $x$ & & & WET & & $\mathrm{LC} / \mathrm{LC} / \mathrm{LC}$ & \\
\hline Chloroceryle americana (Gmelin, 1788) & Green Kingfisher & & $x$ & & WET & & $\mathrm{LC} / \mathrm{LC} / \mathrm{LC}$ & \\
\hline \multicolumn{9}{|l|}{ Momotidae } \\
\hline Baryphthengus ruficapillus (Vieillot, 1818) ATL & Rufous-capped Motmot & $x$ & & & SEM & & $\mathrm{LC} / \mathrm{LC} / \mathrm{LC}$ & \\
\hline \multicolumn{9}{|l|}{ GALBULIFORMES } \\
\hline \multicolumn{9}{|l|}{ Galbulidae } \\
\hline Galbula ruficauda Cuvier, 1816 & Rufous-tailed Jacamar & $x$ & & & UBQ & & $\mathrm{LC} / \mathrm{LC} / \mathrm{LC}$ & \\
\hline \multicolumn{9}{|l|}{ Bucconidae } \\
\hline Nystalus chacuru (Vieillot, 1816) & White-eared Puffbird & $x$ & & & REG & & $\mathrm{LC} / \mathrm{LC} / \mathrm{LC}$ & \\
\hline \multicolumn{9}{|l|}{ PICIFORMES } \\
\hline \multicolumn{9}{|l|}{ Ramphastidae } \\
\hline Ramphastos toco Statius Müller, 1776 & Toco Toucan & $x$ & & & UBQ & & $\mathrm{LC} / \mathrm{LC} / \mathrm{LC}$ & $x$ \\
\hline \multicolumn{9}{|l|}{ Picidae } \\
\hline Picumnus albosquamatus d'Orbigny, 1840 & White-wedged Piculet & $x$ & & & UBQ & & $\mathrm{LC} / \mathrm{LC} / \mathrm{LC}$ & \\
\hline Melanerpes candidus (Otto, 1796) & White Woodpecker & $x$ & & & UBQ & & $\mathrm{LC} / \mathrm{LC} / \mathrm{LC}$ & \\
\hline Veniliornis passerinus (Linnaeus, 1766) & Little Woodpecker & $x$ & & & UBQ & & $\mathrm{LC} / \mathrm{LC} / \mathrm{LC}$ & \\
\hline Colaptes melanochloros (Gmelin, 1788) & Green-barred Woodpecker & $x$ & & & UBQ & & $\mathrm{LC} / \mathrm{LC} / \mathrm{LC}$ & \\
\hline Colaptes campestris (Vieillot, 1818) & Campo Flicker & $x$ & & & REG & & $\mathrm{LC} / \mathrm{LC} / \mathrm{LC}$ & \\
\hline Dryocopus lineatus (Linnaeus, 1766) & Lineated Woodpecker & $x$ & & & REG & & $\mathrm{LC} / \mathrm{LC} / \mathrm{LC}$ & \\
\hline \multicolumn{9}{|l|}{ CARIAMIFORMES } \\
\hline \multicolumn{9}{|l|}{ Cariamidae } \\
\hline Cariama cristata (Linnaeus, 1766) & Red-legged Seriema & $x$ & & & REG & & $\mathrm{LC} / \mathrm{LC} / \mathrm{LC}$ & \\
\hline \multicolumn{9}{|l|}{ FALCONIFORMES } \\
\hline \multicolumn{9}{|l|}{ Falconidae } \\
\hline Caracara plancus (Miller, 1777) & Southern Caracara & $x$ & & & REG & & $\mathrm{LC} / \mathrm{LC} / \mathrm{LC}$ & \\
\hline Milvago chimachima (Vieillot, 1816) & Yellow-headed Caracara & $x$ & & & REG & & $\mathrm{LC} / \mathrm{LC} / \mathrm{LC}$ & \\
\hline Herpetotheres cachinnans (Linnaeus, 1758) & Laughing Falcon & $x$ & & & REG & & $\mathrm{LC} / \mathrm{LC} / \mathrm{LC}$ & \\
\hline Micrastur semitorquatus (Vieillot, 1817) & Collared Forest-Falcon & $x$ & & & SAV & & $\mathrm{LC} / \mathrm{LC} / \mathrm{LC}$ & \\
\hline Falco sparverius Linnaeus, 1758 & American Kestrel & & $x$ & & REG & & $\mathrm{LC} / \mathrm{LC} / \mathrm{LC}$ & \\
\hline \multicolumn{9}{|l|}{ PSITTACIFORMES } \\
\hline \multicolumn{9}{|l|}{ Psittacidae } \\
\hline Primolius maracana (Vieillot, 1816) & Blue-winged Macaw & $x$ & & & SEM & & NT/NT/VU & $x$ \\
\hline Psittacara leucophthalmus (Statius Müller, 1776) & White-eyed Parakeet & $x$ & & & UBQ & & $\mathrm{LC} / \mathrm{LC} / \mathrm{LC}$ & $x$ \\
\hline Forpus xanthopterygius (Spix, 1824) & Blue-winged Parrotlet & $x$ & & & UBQ & & $\mathrm{LC} / \mathrm{LC} / \mathrm{LC}$ & $x$ \\
\hline Brotogeris chiriri (Vieillot, 1818) & Yellow-chevroned Parakeet & $x$ & & & UBQ & & LC/ LC/ LC & $x$ \\
\hline Pionus maximiliani (Kuhl, 1820) & Scaly-headed Parrot & $x$ & & & SEM & & $\mathrm{LC} / \mathrm{LC} / \mathrm{LC}$ & $x$ \\
\hline Amazona aestiva (Linnaeus, 1758) & Turquoise-fronted Parrot & $x$ & & & SEM & & $\mathrm{LC} / \mathrm{NT} / \mathrm{NT}$ & $x$ \\
\hline \multicolumn{9}{|l|}{ PASSERIFORMES } \\
\hline Thamnophilidae & & & & & & & & \\
\hline Herpsilochmus atricapillus Pelzeln, 1868 & Black-capped Antwren & $x$ & & & SAV/SEM & & $\mathrm{LC} / \mathrm{LC} / \mathrm{LC}$ & \\
\hline Herpsilochmus longirostris Pelzeln, 1868 CER & Large-billed Antwren & $x$ & & & SEM & & $\mathrm{LC} / \mathrm{LC} / \mathrm{NT}$ & \\
\hline Thamnophilus doliatus (Linnaeus, 1764) & Barred Antshrike & $x$ & & & UBQ & & $\mathrm{LC} / \mathrm{LC} / \mathrm{LC}$ & \\
\hline Thamnophilus pelzelni Hellmayr, 1924 & Planalto Slaty-Antshrike & $x$ & & & CER & & LC/ LC/ LC & \\
\hline Taraba major (Vieillot, 1816) & Great Antshrike & $x$ & & & SAV/SEM & & $\mathrm{LC} / \mathrm{LC} / \mathrm{LC}$ & \\
\hline Conopophagidae & & & & & & & & \\
\hline Conopophaga lineata (Wied, 1831) ATL & Rufous Gnateater & $x$ & & & SEM & & $\mathrm{LC} / \mathrm{LC} / \mathrm{LC}$ & \\
\hline Dendrocolaptidae & & & & & & & & \\
\hline Sittasomus griseicapillus (Vieillot, 1818) & Olivaceous Woodcreeper & $x$ & & & SEM & & $\mathrm{LC} / \mathrm{LC} / \mathrm{LC}$ & \\
\hline Lepidocolaptes angustirostris (Vieillot, 1818) & Narrow-billed Woodcreeper & $x$ & & & UBQ & & $\mathrm{LC} / \mathrm{LC} / \mathrm{LC}$ & \\
\hline Furnariidae & & & & & & & & \\
\hline Furnarius rufus (Gmelin, 1788) & Rufous Hornero & $x$ & & & UBQ & & $\mathrm{LC} / \mathrm{LC} / \mathrm{LC}$ & \\
\hline Automolus leucophthalmus (Wied, 1821) ATL & White-eyed Foliage-gleaner & $x$ & & & SEM & & $\mathrm{LC} / \mathrm{LC} / \mathrm{LC}$ & \\
\hline Certhiaxis cinnamomeus (Gmelin, 1788) & Yellow-chinned Spinetail & $x$ & & & WET & & $\mathrm{LC} / \mathrm{LC} / \mathrm{LC}$ & \\
\hline Synallaxis frontalis Pelzeln, 1859 & Sooty-fronted Spinetail & $x$ & & & REG & & $\mathrm{LC} / \mathrm{LC} / \mathrm{LC}$ & \\
\hline Synallaxis albescens Temminck, 1823 & Pale-breasted Spinetail & & $x$ & & CER & & LC/ LC / LC & \\
\hline Pipridae & & & & & & & & \\
\hline Neopelma pallescens (Lafresnaye, 1853) & Pale-bellied Tyrant-Manakin & $x$ & & & CER & & $\mathrm{LC} / \mathrm{LC} / \mathrm{NT}$ & \\
\hline Antilophia galeata (Lichtenstein, 1823) СЕR & Helmeted Manakin & $x$ & & & CER/SEM & & $\mathrm{LC} / \mathrm{LC} / \mathrm{LC}$ & \\
\hline Tityridae & & & & & & & & \\
\hline Pachyramphus viridis (Vieillot, 1816) & Green-backed Becard & $x$ & & & REG & & $\mathrm{LC} / \mathrm{LC} / \mathrm{LC}$ & \\
\hline Pachyramphus validus (Lichtenstein, 1823) & Crested Becard & $x$ & & & REG & MPR & $\mathrm{LC} / \mathrm{LC} / \mathrm{LC}$ & \\
\hline
\end{tabular}




\begin{tabular}{|c|c|c|c|c|c|c|c|c|}
\hline Taxon & English name & Quan. & Qual. & Data. & Phys. & Mig. & $\begin{array}{l}\text { Conserv. } \\
\text { status }\end{array}$ & Traf. \\
\hline \multicolumn{9}{|l|}{ Platyrinchidae } \\
\hline Platyrinchus mystaceus Vieillot, 1818 & White-throated Spadebill & $x$ & & & SEM & & $\mathrm{LC} / \mathrm{LC} / \mathrm{LC}$ & \\
\hline \multicolumn{9}{|l|}{ Rhynchocyclidae } \\
\hline Leptopogon amaurocephalus Tschudi, 1846 & Sepia-capped Flycatcher & $\mathrm{x}$ & & & SEM & & $\mathrm{LC} / \mathrm{LC} / \mathrm{LC}$ & \\
\hline Corythopis delalandi Tschudi, 1844 & Ringed Antpipit & $\mathrm{x}$ & & & SEM & & $\mathrm{LC} / \mathrm{LC} / \mathrm{LC}$ & \\
\hline Tolmomyias sulphurescens (Spix, 1825) & Yellow-olive Flycatcher & & $\mathrm{x}$ & & SEM & & $\mathrm{LC} / \mathrm{LC} / \mathrm{LC}$ & \\
\hline Todirostrum poliocephalum (Wied, 1831) ATL & Gray-headed Tody-Flycatcher & $x$ & & & SEM & & $\mathrm{LC} / \mathrm{LC} / \mathrm{LC}$ & \\
\hline Todirostrum cinereum (Linnaeus, 1766) & Common Tody-Flycatcher & $\mathrm{x}$ & & & UBQ & & $\mathrm{LC} / \mathrm{LC} / \mathrm{LC}$ & \\
\hline Poecilotriccus latirostris (Pelzeln, 1868) & Rusty-fronted Tody-Flycatcher & $\mathrm{x}$ & & & CER & & $\mathrm{LC} / \mathrm{LC} / \mathrm{LC}$ & \\
\hline $\begin{array}{l}\text { Hemitriccus margaritaceiventer (d'Orbigny \& } \\
\text { Lafresnaye, 1837) }\end{array}$ & Pearly-vented Tody-tyrant & $x$ & & & CER & & $\mathrm{LC} / \mathrm{LC} / \mathrm{LC}$ & \\
\hline \multicolumn{9}{|l|}{ Tyrannidae } \\
\hline Hirundinea ferruginea (Gmelin, 1788) & Cliff Flycatcher & $\mathrm{x}$ & & & REG & & LC/ LC/ LC & \\
\hline Camptostoma obsoletum (Temminck, 1824) & Southern Beardless-Tyrannulet & $\mathrm{x}$ & & & UBQ & & $\mathrm{LC} / \mathrm{LC} / \mathrm{LC}$ & \\
\hline Elaenia flavogaster (Thunberg, 1822) & Yellow-bellied Elaenia & $\mathrm{x}$ & & & UBQ & & $\mathrm{LC} / \mathrm{LC} / \mathrm{LC}$ & \\
\hline Elaenia mesoleuca (Deppe, 1830) & Olivaceous Elaenia & $\mathrm{x}$ & & & REG & & LC/ LC/ LC & \\
\hline Elaenia obscura (d'Orbigny \& Lafresnaye, 1837) & Highland Elaenia & $\mathrm{x}$ & & & CER & & $\mathrm{LC} / \mathrm{LC} / \mathrm{LC}$ & \\
\hline Myiopagis viridicata (Vieillot, 1817) & Greenish Elaenia & $\mathrm{x}$ & & & SEM & MPR & $\mathrm{LC} / \mathrm{LC} / \mathrm{LC}$ & \\
\hline Phaeomyias murina (Spix, 1825) & Mouse-colored Tyrannulet & $\mathrm{x}$ & & & CER & & LC/ LC/ LC & \\
\hline Serpophaga subcristata (Vieillot, 1817) & White-crested Tyrannulet & $\mathrm{x}$ & & & UBQ & & $\mathrm{LC} / \mathrm{LC} / \mathrm{LC}$ & \\
\hline Legatus leucophaius (Vieillot, 1818) & Piratic Flycatcher & $\mathrm{x}$ & & & REG & MPR & $\mathrm{LC} / \mathrm{LC} / \mathrm{LC}$ & \\
\hline Myiarchus swainsoni Cabanis \& Heine, 1859 & Swainson's Flycatcher & $\mathrm{x}$ & & & UBQ & MPR & $\mathrm{LC} / \mathrm{LC} / \mathrm{LC}$ & \\
\hline Myiarchus ferox (Gmelin, 1789) & Short-crested Flycatcher & $\mathrm{x}$ & & & UBQ & & $\mathrm{LC} / \mathrm{LC} / \mathrm{LC}$ & \\
\hline Sirystes sibilator (Vieillot, 1818) & Sibilant Sirystes & $\mathrm{x}$ & & & REG & & $\mathrm{LC} / \mathrm{LC} / \mathrm{LC}$ & \\
\hline Casiornis rufus (Vieillot, 1816) & Rufous Casiornis & $\mathrm{x}$ & & & REG & MPR & $\mathrm{LC} / \mathrm{LC} / \mathrm{LC}$ & \\
\hline Pitangus sulphuratus (Linnaeus, 1766) & Great Kiskadee & $\mathrm{x}$ & & & UBQ & MPR & $\mathrm{LC} / \mathrm{LC} / \mathrm{LC}$ & \\
\hline Myiodynastes maculatus (Statius Müller, 1776) & Streaked Flycatcher & $\mathrm{x}$ & & & UBQ & MPR & $\mathrm{LC} / \mathrm{LC} / \mathrm{LC}$ & \\
\hline Megarynchus pitangua (Linnaeus, 1766) & Boat-billed Flycatcher & $\mathrm{x}$ & & & UBQ & & $\mathrm{LC} / \mathrm{LC} / \mathrm{LC}$ & \\
\hline Myiozetetes similis (Spix, 1825) & Social Flycatcher & $\mathrm{x}$ & & & UBQ & & $\mathrm{LC} / \mathrm{LC} / \mathrm{LC}$ & \\
\hline Tyrannus melancholicus Vieillot, 1819 & Tropical Kingbird & $\mathrm{x}$ & & & UBQ & MPR & $\mathrm{LC} / \mathrm{LC} / \mathrm{LC}$ & \\
\hline Tyrannus savana Daudin, 1802 & Fork-tailed Flycatcher & $\mathrm{x}$ & & & REG/CER & MPR & $\mathrm{LC} / \mathrm{LC} / \mathrm{LC}$ & \\
\hline $\begin{array}{l}\text { Griseotyrannus aurantioatrocristatus (d'Orbigny } \\
\text { \& Lafresnaye, 1837) }\end{array}$ & Crowned Slaty Flycatcher & $x$ & & & REG & MPR & $\mathrm{LC} / \mathrm{LC} / \mathrm{LC}$ & \\
\hline Empidonomus varius (Vieillot, 1818) & Variegated Flycatcher & $\mathrm{x}$ & & & REG/CER & MPR & $\mathrm{LC} / \mathrm{LC} / \mathrm{LC}$ & \\
\hline Colonia colonus (Vieillot, 1818) & Long-tailed Tyrant & $\mathrm{x}$ & & & & & $\mathrm{LC} / \mathrm{LC} / \mathrm{LC}$ & \\
\hline Myiophobus fasciatus (Statius Müller, 1776) & Bran-colored Flycatcher & $\mathrm{x}$ & & & SEM & MPR & $\mathrm{LC} / \mathrm{LC} / \mathrm{LC}$ & \\
\hline Pyrocephalus rubinus (Boddaert, 1783) & Vermilion Flycatcher & $\mathrm{x}$ & & & REG/CER & MPR & $\mathrm{LC} / \mathrm{LC} / \mathrm{LC}$ & \\
\hline Fluvicola nengeta (Linnaeus, 1766) & Masked Water-Tyrant & $\mathrm{x}$ & & & WET & & $\mathrm{LC} / \mathrm{LC} / \mathrm{LC}$ & \\
\hline Gubernetes yetapa (Vieillot, 1818) & Streamer-tailed Tyrant & $\mathrm{x}$ & & & WET & & $\mathrm{LC} / \mathrm{LC} / \mathrm{LC}$ & \\
\hline Cnemotriccus fuscatus (Wied, 1831) & Fuscous Flycatcher & $\mathrm{x}$ & & & REG/CER & & $\mathrm{LC} / \mathrm{LC} / \mathrm{LC}$ & \\
\hline Lathrotriccus euleri (Cabanis, 1868) & Euler's Flycatcher & $\mathrm{x}$ & & & REG/CER & MPR & $\mathrm{LC} / \mathrm{LC} / \mathrm{LC}$ & \\
\hline Xolmis cinereus (Vieillot, 1816) & Gray Monjita & $\mathrm{x}$ & & & REG & & $\mathrm{LC} / \mathrm{LC} / \mathrm{LC}$ & \\
\hline Xolmis velatus (Lichtenstein, 1823) & White-rumped Monjita & $\mathrm{x}$ & & & REG & & LC/ LC/ LC & \\
\hline \multicolumn{9}{|l|}{ Vireonidae } \\
\hline Cyclarhis gujanensis (Gmelin, 1789) & Rufous-browed Peppershrike & $\mathrm{x}$ & & & UBQ & & $\mathrm{LC} / \mathrm{LC} / \mathrm{LC}$ & \\
\hline Hylophilus amaurocephalus (Nordmann, 1835) & Gray-eyed Greenlet & $\mathrm{x}$ & & & CER & & $\mathrm{LC} / \mathrm{LC} / \mathrm{LC}$ & \\
\hline Vireo chivi (Vieillot, 1817) & Chivi Vireo & $\mathrm{x}$ & & & UBQ & MPR & $\mathrm{LC} / \mathrm{LC} / \mathrm{LC}$ & \\
\hline \multicolumn{9}{|l|}{ Corvidae } \\
\hline Cyanocorax cristatellus (Temminck, 1823) ${ }^{\mathrm{CER}}$ & Curl-crested Jay & $x$ & & & CER & & $\mathrm{LC} / \mathrm{LC} / \mathrm{LC}$ & $x$ \\
\hline Cyanocorax chrysops (Vieillot, 1818) & Plush-crested Jay & $\mathrm{x}$ & & & UBQ & & $\mathrm{LC} / \mathrm{LC} / \mathrm{LC}$ & $\mathrm{x}$ \\
\hline \multicolumn{9}{|l|}{ Hirundinidae } \\
\hline Pygochelidon cyanoleuca (Vieillot, 1817) & Blue-and-white Swallow & $\mathrm{x}$ & & & UBQ & & $\mathrm{LC} / \mathrm{LC} / \mathrm{LC}$ & \\
\hline Stelgidopteryx ruficollis (Vieillot, 1817) & $\begin{array}{l}\text { Southern Rough-winged } \\
\text { Swallow }\end{array}$ & $\mathrm{x}$ & & & REG & MPR & $\mathrm{LC} / \mathrm{LC} / \mathrm{LC}$ & \\
\hline Progne tapera (Vieillot, 1817) & Brown-chested Martin & $\mathrm{x}$ & & & REG & MPR & $\mathrm{LC} / \mathrm{LC} / \mathrm{LC}$ & \\
\hline Progne chalybea (Gmelin, 1789) & Gray-breasted Martin & $\mathrm{x}$ & & & REG & MPR & $\mathrm{LC} / \mathrm{LC} / \mathrm{LC}$ & \\
\hline \multicolumn{9}{|l|}{ Troglodytidae } \\
\hline Troglodytes musculus Naumann, 1823 & Southern House Wren & $\mathrm{x}$ & & & REG & & $\mathrm{LC} / \mathrm{LC} / \mathrm{LC}$ & \\
\hline \multicolumn{9}{|l|}{ Donacobiidae } \\
\hline Donacobius atricapilla (Linnaeus, 1766) & Black-capped Donacobius & $x$ & & & WET & & $\mathrm{LC} / \mathrm{LC} / \mathrm{LC}$ & \\
\hline \multicolumn{9}{|l|}{ Turdidae } \\
\hline Turdus leucomelas Vieillot, 1818 & Pale-breasted Thrush & $\mathrm{x}$ & & & UBQ & & $\mathrm{LC} / \mathrm{LC} / \mathrm{LC}$ & $\mathrm{x}$ \\
\hline
\end{tabular}




\begin{tabular}{|c|c|c|c|c|c|c|c|c|}
\hline Taxon & English name & Quan. & Qual. & Data. & Phys. & Mig. & $\begin{array}{c}\text { Conserv. } \\
\text { status }\end{array}$ & Traf. \\
\hline Turdus rufiventris Vieillot, 1818 & Rufous-bellied Thrush & $\mathrm{X}$ & & & SEM & & LC/ LC/ LC & $\mathrm{x}$ \\
\hline Turdus amaurochalinus Cabanis, 1850 & Creamy-bellied Thrush & $x$ & & & UBQ & & $\mathrm{LC} / \mathrm{LC} / \mathrm{LC}$ & $x$ \\
\hline Turdus subalaris (Seebohm, 1887) ATL & Eastern Slaty Thrush & $\mathrm{x}$ & & & SEM & MPR & $\mathrm{LC} / \mathrm{LC} / \mathrm{LC}$ & \\
\hline Turdus albicollis Vieillot, 1818 & White-necked Thrush & $x$ & & & SEM & MPR & $\mathrm{LC} / \mathrm{LC} / \mathrm{LC}$ & $x$ \\
\hline \multicolumn{9}{|l|}{ Mimidae } \\
\hline Mimus saturninus (Lichtenstein, 1823) & Chalk-browed Mockingbird & $x$ & & & REG & & $\mathrm{LC} / \mathrm{LC} / \mathrm{LC}$ & \\
\hline \multicolumn{9}{|l|}{ Passerellidae } \\
\hline Zonotrichia capensis (Statius Müller, 1776) & Rufous-collared Sparrow & $\mathrm{x}$ & & & REG & & $\mathrm{LC} / \mathrm{LC} / \mathrm{LC}$ & $x$ \\
\hline Ammodramus humeralis (Bosc, 1792) & Grassland Sparrow & $x$ & & & REG & & LC/ LC/ LC & $x$ \\
\hline Arremon flavirostris Swainson, 1838 & Saffron-billed Sparrow & $x$ & & & SEM & & $\mathrm{LC} / \mathrm{LC} / \mathrm{LC}$ & $\mathrm{x}$ \\
\hline \multicolumn{9}{|l|}{ Parulidae } \\
\hline Setophaga pitiayumi (Vieillot, 1817) & Tropical Parula & $x$ & & & SEM & & $\mathrm{LC} / \mathrm{LC} / \mathrm{LC}$ & \\
\hline Geothlypis aequinoctialis (Gmelin, 1789) & Masked Yellowthroat & $x$ & & & WET & & LC/ LC/ LC & \\
\hline Basileuterus culicivorus (Deppe, 1830) & Golden-crowned Warbler & $x$ & & & UBQ & & LC/ LC/ LC & \\
\hline Myiothlypis flaveola Baird, 1865 & Flavescent Warbler & $x$ & & & UBQ & & LC/ LC/ LC & \\
\hline \multicolumn{9}{|l|}{ Icteridae } \\
\hline Cacicus haemorrhous (Linnaeus, 1766) & Red-rumped Cacique & $x$ & & & SEM & & LC/ LC/ LC & $x$ \\
\hline Icterus pyrrhopterus (Vieillot, 1819) & Variable Oriole & $x$ & & & REG & & $\mathrm{LC} / \mathrm{LC} / \mathrm{LC}$ & $x$ \\
\hline Gnorimopsar chopi (Vieillot, 1819) & Chopi Blackbird & $x$ & & & REG & & $\mathrm{LC} / \mathrm{LC} / \mathrm{LC}$ & $\mathrm{x}$ \\
\hline Amblyramphus holosericeus (Scopoli, 1786) & Scarlet-headed Blackbird & $x$ & & & WET & & $\mathrm{LC} / \mathrm{LC} / \mathrm{LC}$ & \\
\hline Chrysomus ruficapillus (Vieillot, 1819) & Chestnut-capped Blackbird & $x$ & & & WET & & LC/ LC/ LC & $x$ \\
\hline Pseudoleistes guirahuro (Vieillot, 1819) & Yellow-rumped Marshbird & $x$ & & & REG/WET & & $\mathrm{LC} / \mathrm{LC} / \mathrm{LC}$ & $x$ \\
\hline Molothrus bonariensis (Gmelin, 1789) & Shiny Cowbird & $\mathrm{x}$ & & & REG & & $\mathrm{LC} / \mathrm{LC} / \mathrm{LC}$ & $x$ \\
\hline \multicolumn{9}{|l|}{ Thraupidae } \\
\hline Pipraeidea melanonota (Vieillot, 1819) & Fawn-breasted Tanager & $x$ & & & SEM & & LC/ LC/ LC & \\
\hline Schistochlamys melanopis (Latham, 1790) & Black-faced Tanager & $x$ & & & REG & & $\mathrm{LC} / \mathrm{LC} / \mathrm{LC}$ & $x$ \\
\hline Schistochlamys ruficapillus (Vieillot, 1817) & Cinnamon Tanager & $x$ & & & REG & & $\mathrm{LC} / \mathrm{LC} / \mathrm{LC}$ & $\mathrm{x}$ \\
\hline Paroaria capitata (d'Orbigny \& Lafresnaye, 1837) & Yellow-billed Cardinal & $x$ & & & REG & & $\mathrm{LC} / \mathrm{LC} / \mathrm{LC}$ & $x$ \\
\hline Tangara sayaca (Linnaeus, 1766) & Sayaca Tanager & $x$ & & & REG & & $\mathrm{LC} / \mathrm{LC} / \mathrm{LC}$ & $x$ \\
\hline Tangara palmarum (Wied, 1821) & Palm Tanager & $x$ & & & UBQ & & LC/ LC/ LC & $x$ \\
\hline Tangara cayana (Linnaeus, 1766) & Burnished-buff Tanager & $x$ & & & UBQ & & $\mathrm{LC} / \mathrm{LC} / \mathrm{LC}$ & $x$ \\
\hline Nemosia pileata (Boddaert, 1783) & Hooded Tanager & $x$ & & & UBQ & & $\mathrm{LC} / \mathrm{LC} / \mathrm{LC}$ & \\
\hline Conirostrum speciosum (Temminck, 1824) & Chestnut-vented Conebill & $x$ & & & UBQ & & $\mathrm{LC} / \mathrm{LC} / \mathrm{LC}$ & \\
\hline Sicalis flaveola (Linnaeus, 1766) & Saffron Finch & $x$ & & & REG & & $\mathrm{LC} / \mathrm{LC} / \mathrm{LC}$ & $x$ \\
\hline Hemithraupis guira (Linnaeus, 1766) & Guira Tanager & $x$ & & & SEM & & $\mathrm{LC} / \mathrm{LC} / \mathrm{LC}$ & \\
\hline Volatinia jacarina (Linnaeus, 1766) & Blue-black Grassquit & $x$ & & & REG & & $\mathrm{LC} / \mathrm{LC} / \mathrm{LC}$ & $x$ \\
\hline Eucometis penicillata (Spix, 1825) & Gray-headed Tanager & $x$ & & & SEM & & $\mathrm{LC} / \mathrm{LC} / \mathrm{LC}$ & \\
\hline Trichothraupis melanops (Vieillot, 1818) & Black-goggled Tanager & $x$ & & & SEM & & LC/ LC/ LC & \\
\hline Coryphospingus cucullatus (Statius Müller, 1776) & Red-crested Finch & $x$ & & & REG & & $\mathrm{LC} / \mathrm{LC} / \mathrm{LC}$ & $x$ \\
\hline Tachyphonus coronatus (Vieillot, 1822) ATL & Ruby-crowned Tanager & $x$ & & & SEM & & $\mathrm{LC} / \mathrm{LC} / \mathrm{LC}$ & $x$ \\
\hline Ramphocelus carbo (Pallas, 1764) & Silver-beaked Tanager & $x$ & & & SEM/CER & & $\mathrm{LC} / \mathrm{LC} / \mathrm{LC}$ & $x$ \\
\hline Tersina viridis (Illiger, 1811) & Swallow Tanager & $x$ & & & UBQ & MPR & LC/ LC/ LC & \\
\hline Dacnis cayana (Linnaeus, 1766) & Blue Dacnis & $x$ & & & UBQ & & $\mathrm{LC} / \mathrm{LC} / \mathrm{LC}$ & $x$ \\
\hline Coereba flaveola (Linnaeus, 1758) & Bananaquit & $\mathrm{x}$ & & & UBQ & & $\mathrm{LC} / \mathrm{LC} / \mathrm{LC}$ & \\
\hline Sporophila lineola (Linnaeus, 1758) & Lined Seedeater & $x$ & & & REG & MPR & $\mathrm{LC} / \mathrm{LC} / \mathrm{LC}$ & $x$ \\
\hline Sporophila caerulescens (Vieillot, 1823) & Double-collared Seedeater & $\mathrm{x}$ & & & REG & MPR & LC/ LC/ LC & $x$ \\
\hline Sporophila leucoptera (Vieillot, 1817) & White-bellied Seedeater & $x$ & & & WET & & $\mathrm{LC} / \mathrm{LC} / \mathrm{LC}$ & $x$ \\
\hline Sporophila angolensis (Linnaeus, 1766) & Chestnut-bellied Seed-Finch & $x$ & & & REG & & $\mathrm{LC} / \mathrm{LC} / \mathrm{LC}$ & $x$ \\
\hline Emberizoides herbicola (Vieillot, 1817) & Wedge-tailed Grass-Finch & $x$ & & & REG & & $\mathrm{LC} / \mathrm{LC} / \mathrm{LC}$ & \\
\hline Saltatricula atricollis (Vieillot, 1817) CER & Black-throated Saltator & $x$ & & & REG & & LC/ LC/ LC & $x$ \\
\hline Saltator similis d'Orbigny \& Lafresnaye, 1837 & Green-winged Saltator & $x$ & & & SEM & & $\mathrm{LC} / \mathrm{LC} / \mathrm{LC}$ & $x$ \\
\hline \multicolumn{9}{|l|}{ Fringillidae } \\
\hline Spinus magellanicus (Vieillot, 1805) & Hooded Siskin & $x$ & & & REG & & $\mathrm{LC} / \mathrm{LC} / \mathrm{LC}$ & $x$ \\
\hline Euphonia chlorotica (Linnaeus, 1766) & Purple-throated Euphonia & $x$ & & & UBQ & & LC/ LC/ LC & $x$ \\
\hline Euphonia violacea (Linnaeus, 1758) & Violaceous Euphonia & $x$ & & & SEM & & $\mathrm{LC} / \mathrm{LC} / \mathrm{LC}$ & $x$ \\
\hline \multicolumn{9}{|l|}{ Estrildidae } \\
\hline Estrilda astrild (Linnaeus, 1758) EX & Common Waxbill & $x$ & & & REG & & $\mathrm{LC} / \mathrm{LC} / \mathrm{LC}$ & \\
\hline \multicolumn{9}{|l|}{ Passeridae } \\
\hline Passer domesticus (Linnaeus, 1758) EX & House Sparrow & $x$ & & & REG & & LC/ LC/ LC & \\
\hline
\end{tabular}




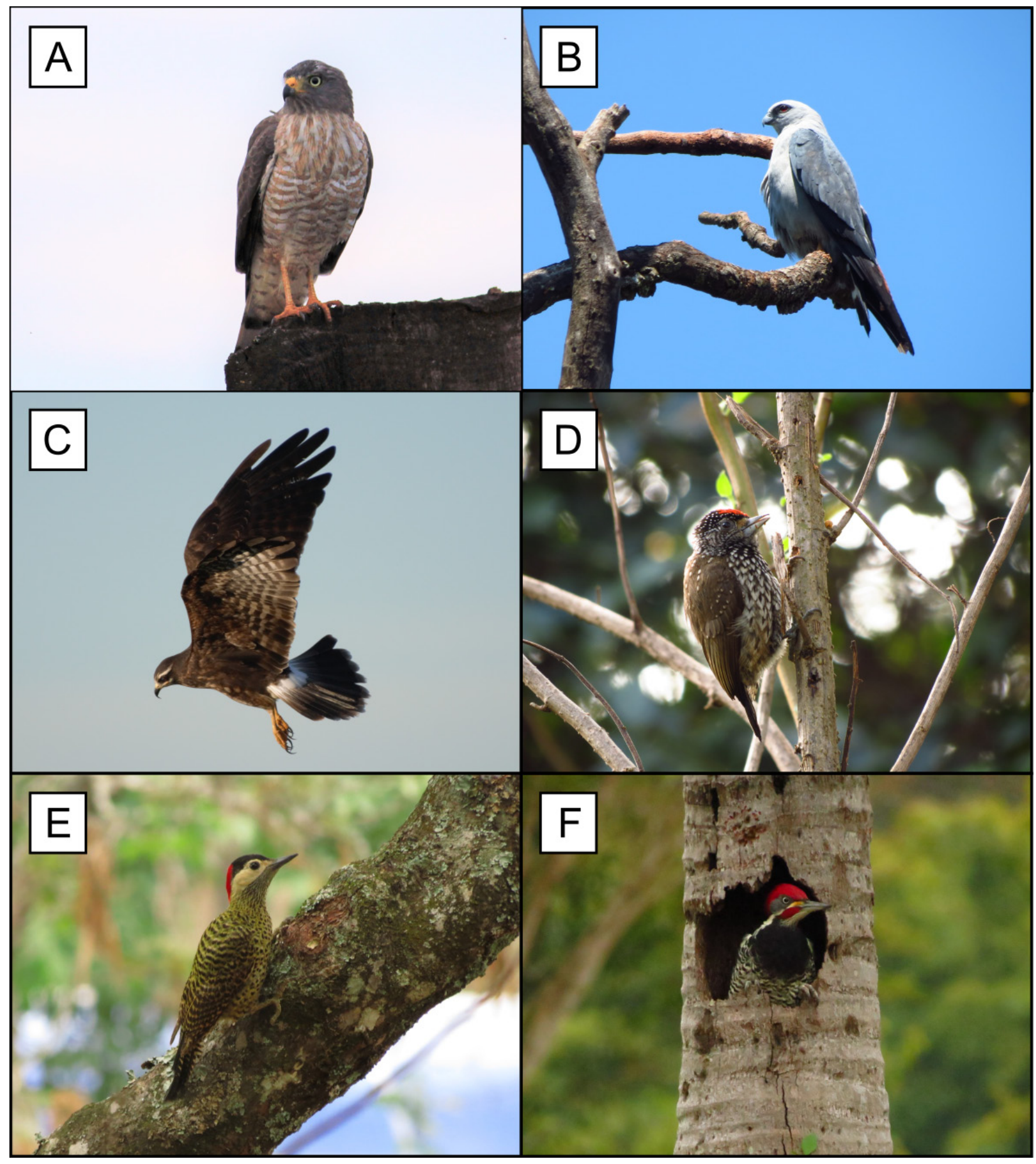

Figure 4. Birds recorded during our survey at the JBMB. A. Roadside Hawk Rupornis magnirostris (point no. 16). B. Plumbeous Kite Ictinea plumbea (point no. 19). C. Snail Kite Rostrhamus sociabilis (point no. 14). D. White-wedged Piculet Picumnus albosquamatus (point no. 06) . E. Green-barred Woodpecker Colaptes melanochloros (point no. 22). F. Lineated Woodpecker Dryocopus lineatus (point no. 19).

\section{Species endemic to the Atlantic Forest}

Thalurania glaucopis (Gmelin, 1788). This species inhabits primary forest, forest edges, scrub, and suburban areas, such as parks and gardens (Weller 2018). It is an active nectarivore hummingbird and its presence is associated with the phenology of several plant species. During our surveys, we had only documented this species once, in April 2017, in a Semideciduous Forest area, at point no. 02.

Baryphthengus ruficapillus (Vieillot, 1818). B. ruficapillus is associated with primary and Gallery Forests, and is usually found in pairs or mixed flocks (Snow and Kirwan 2018). We documented it in a Semideciduous Forest near the Vargem Limpa River. Although there is evidence of population decline (BirdLife International 2018), it is abundant in the Semideciduous Forests of the surveyed area. We recorded it at points no. 02, 03, and 04.

Conopophaga lineata (Wied, 1831). The species is mostly associated with Ombrophilous Forest, and is regularly found in well-developed secondary growth and Gallery Forests. In São Paulo state, it is associated with humid 


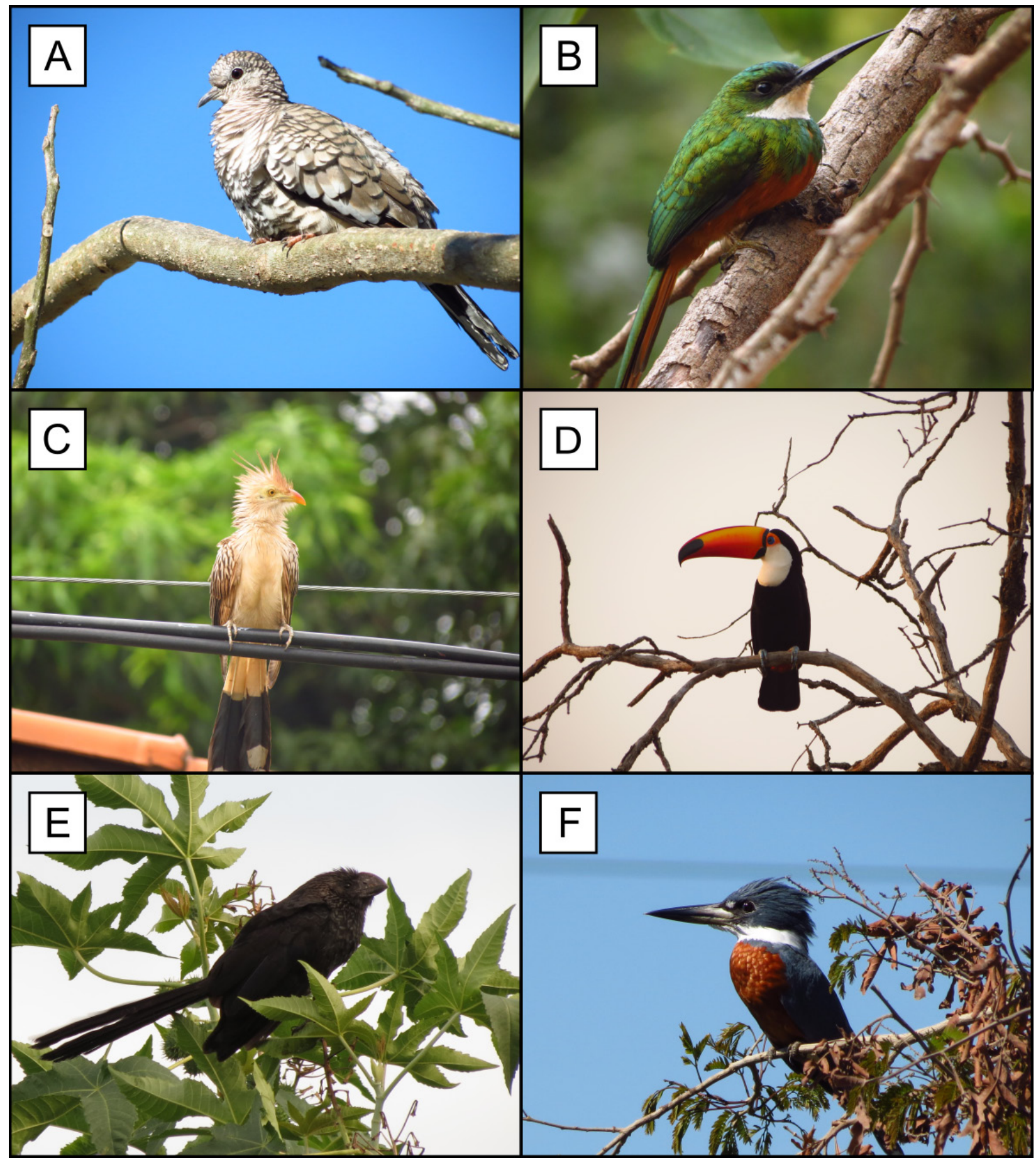

Figure 5. Birds recorded during our survey at the JBMB. A. Scaled Dove Columbina squammata (point no. 19). B. Rufous-tailed Jacamar Galbula ruficauda (point no. 04). C. Guira Cuckoo Guira guira (point no. 01). D. Toco Toucan Ramphastos toco (point no. 12). E. Smooth-billed Ani Crotophaga ani (point no. 22). F. Ringed Kingfisher Megaceryle torquata (point no. 15).

forests and preferentially inhabits bamboo thickets and dense growth (Whitney 2018). In the JBMB, we observed a pair of birds near a nest next to the main trail, which were observed for several months in 2017 at point no. 03.

Automolus leucophthalmus (Wied, 1821). This species inhabits closed and humid areas, and is found in understory branches near the ground up to the midcanopy. $A$. leucophtalmus plays an important role in the formation of mixed flocks, and it is one of the species responsible for the maintenance of group cohesion (Remsen Jr and
Kirwan 2018). It is quite common in Brazil, Paraguay, and Argentina (Sick 1997) and it was observed over several months during our surveys, mostly in the humid areas of the Semideciduous Forest, at points no. 02, 25, and 26.

Todirostrum poliocephalum (Wied, 1831). T. poliocephalum inhabits the canopy (Sick 1997) and the edges of humid forests, adjacent clearings, and even gardens (Walther 2018). It forages near the ground and in the canopy of low-growing trees. It is not dependent on 


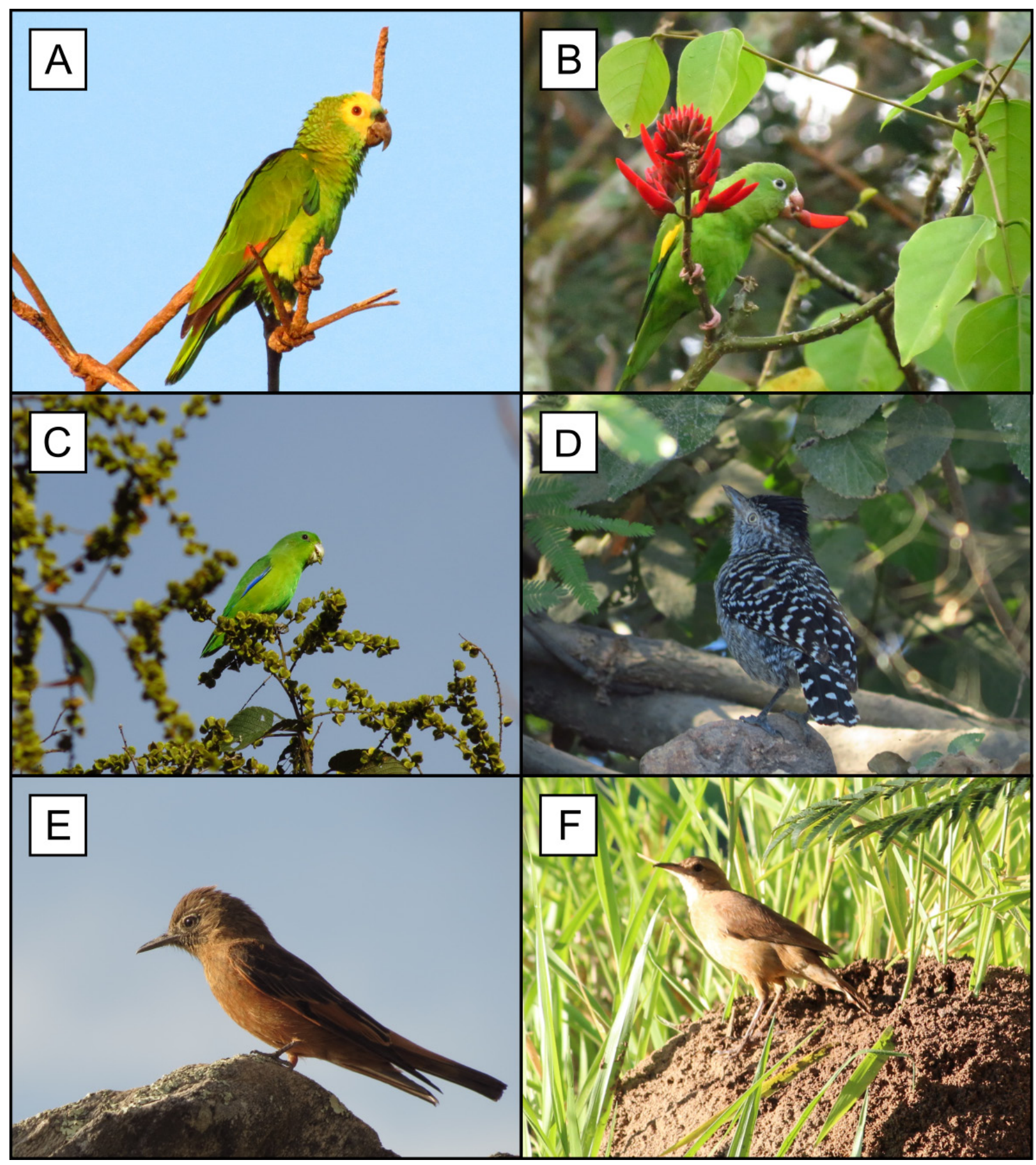

Figure 6. Birds recorded during our survey at the JBMB. A. Turquoise-fronted Parrot Amazona aestiva (point no. 16). B. Yellow-chevroned Parakeet Brotogeris chiriri (point no. 13). C. Blue-winged Parrotlet Forpus xanthopterygius (point no. 17). D. Barred Antshrike Thamnophilus doliatus (point no. 06). E. Cliff Flycatcher Hirundinea ferruginea (point no. 01). F. Rufous Hornero Furnarius rufus (point no. 19).

preserved environments, and it might inhabit humandominated areas with significant tree cover (Sick 1997). We observed it in the JBMB once (December 2016), near flooded fields associated with the Vargem Limpa River. It was recorded at point no. 03 .

Turdus subalaris (Seebohm, 1887). T. subalaris inhabits Subtropical Humid Forests, secondary forests, eucalyptus plantations with understory native vegetation, and Riparian Forests with dense shrub vegetation (Sick 1997). This species is omnivorous, feeding mainly on fruits and supplement its diet with insect prey. It is a migratory species, arriving in southeastern Brazil in mid-September and departing in April (del Hoyo and Collar 2018). In the JBMB, we observed this species in Semideciduous Forest and Forest Savanna during March and April of 2016 and 2017. The species was recorded at points no. 05 and 06 .

Tachyphonus coronatus (Vieillot, 1822). This is a species typical from southeastern Brazil, and inhabits humid forests, clearings, and parks. This species visits 


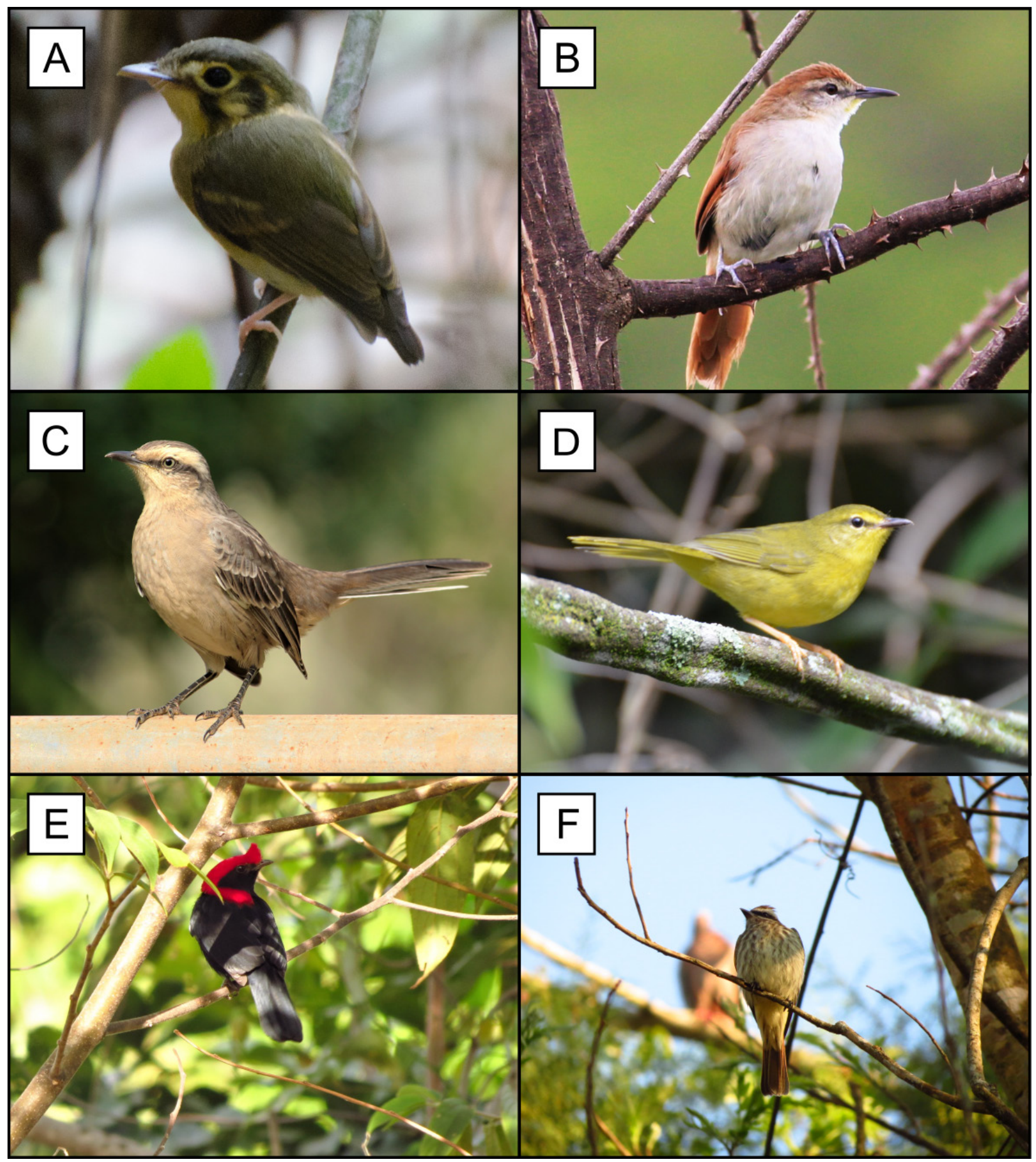

Figure 7. Birds recorded during our survey at the JBMB. A. Eastern White-throated Spadebill Platyrinchus mystaceus (point no. 03). B. Yellow-chinned Spinetail Certhiaxis cinnamomeus (point no. 13). C. Chalk-browed Mockingbird Mimus saturninus (point no. 19). D. Flavescent Warbler Myiothlypis flaveola (point no. 5). E. Helmeted Manakin Antilophia galeata (point no. 08). F. Variegated Flycatcher Empidonomus varius (point no. 18).

fruiting plants and lives near them, avoiding the need for long flights. It forages both in the higher strata and close to the ground (Hilty 2018). This species was observed in the Riparian Forest near the entrance to the main trail and was recorded at point no. 03 .

\section{Cerrado endemic species}

Herpsilochmus longirostris Pelzeln, 1868. This species is found in Bolivia and Brazil, and occupies forests from the understory to canopy in Gallery, Deciduous, and Semideciduous Forests. It is generally found in fragments surrounded by Savanna vegetation. It forages with mixed flocks and exhibits systematical behavior, searching for insects over a whole tree before moving on to the next one. This species is regionally threatened in São Paulo state (São Paulo 2018). In the JBMB, we observed this species in the Semideciduous Flooded Forest at points no. 26 and 27 .

Antilophia galeata (Lichtenstein, 1823). This species is found in Gallery Forests and Savannas, and is 


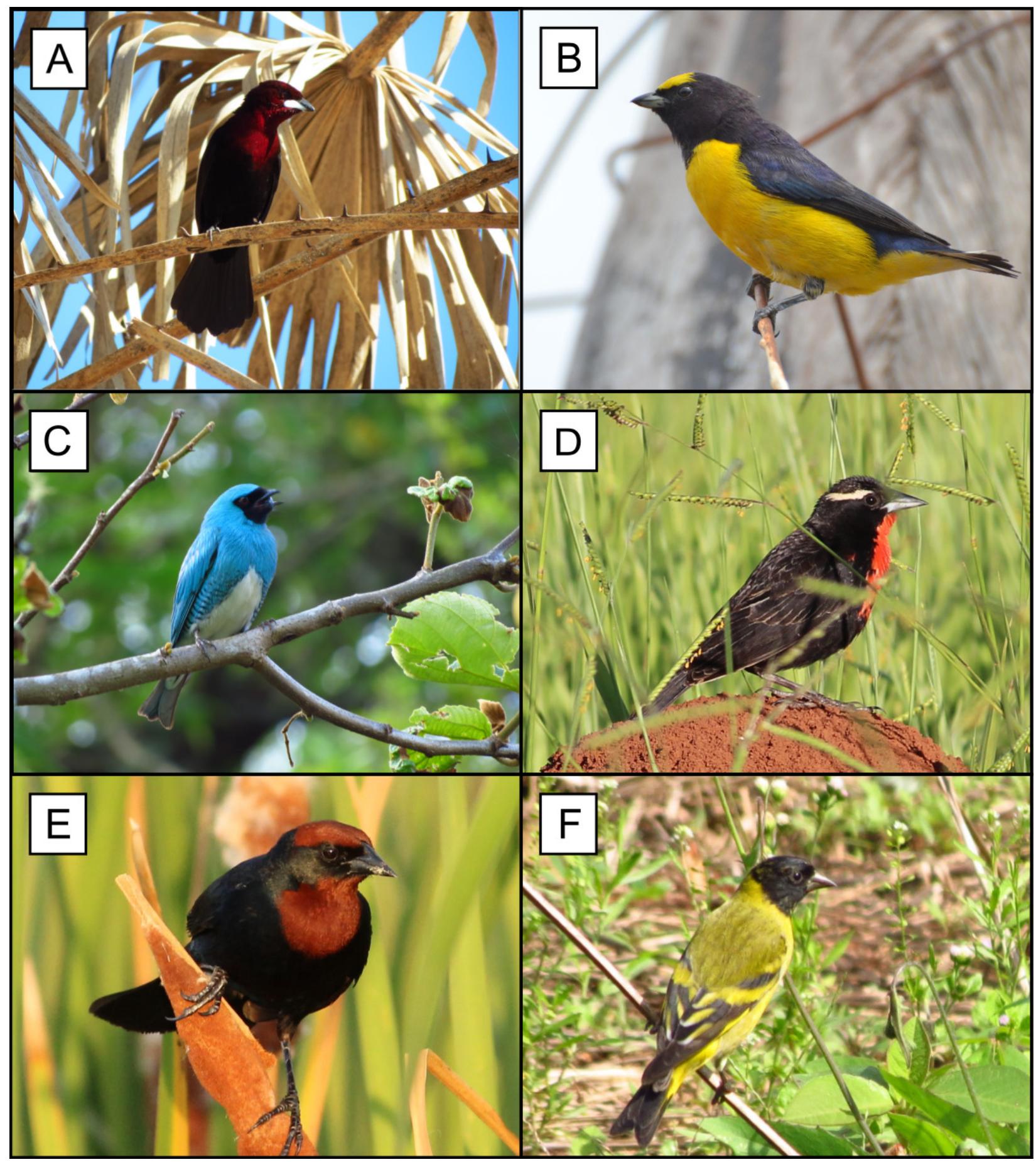

Figure 8. Birds recorded during our survey at the JBMB. A. Silver-beaked Tanager Ramphocelus carbo (point no. 3). B. Purple-throated Euphonia Euphonia chlorotica (point no. 10). C. Swallow Tanager Tersina viridis (point no. 06). D. White-browed Meadowlark Sturnella superciliaris (point no. 16). E. Chestnut-capped Blackbird Chrysomus ruficapillus (point no. 13). F. Hooded Siskin Spinus magellanicus (point no. 19).

common in Deciduous Forests, buriti (Mauritia flexuosa L.f.) palm grooves, and swampy woodland (Snow and de Juana 2018). It has an abundant population in the JBMB, as it is found from primary forests to regeneration areas. It was recorded at points no. 06, 07, 08, and 09.

Cyanocorax cristatellus (Temminck, 1823). C. cristatellus inhabits dry Cerrado forests, but it is also associated with riparian woods, forest edges, and clearing areas with sparse vegetation. It is found in many modified landscapes, including eucalyptus plantations, borders, and gardens (Anjos 2018). Although it is considered endemic to the Cerrado domain, the distribution of this species has expanded to areas of Atlantic Forest (Dário et al. 2002). We observed this species over several months on Forest Savanna edges at point no. 21.

Saltatricula atricollis (Vieillot, 1817). This is a Cerrado-endemic species that possibly also dwells in the Cerrado enclaves in the Pantanal and Caatinga domains (Pacheco 2003). In São Paulo state, it is mainly threatened by fragmentation and the loss of native areas, 
which are transformed into pastures and agricultural areas. This species was found in several different months during our surveys, and was mostly observed in areas undergoing regeneration, near to points no. 20 and 21.

Threatened species. We found 1 species threatened at a global (IUCN 2019), 2 at a national (ICMBIO/MMA 2018), and 8 at a regional level (São Paulo 2018).

Primolius maracana (Vieillot, 1816). Globally and nationally Near Threatened, and regionally Vulnerable. This species lives in Tropical Evergreen Forests, palm groves, and gallery woodlands (Collar et al. 2018a). However, in São Paulo state, the native forest vegetation is restricted to small fragments (Nunes and Galetti 2007), decreasing its area of occurrence. $P$. maracana also has low genetic variability (Craveiro and Miyaki 2000), which further threatens its survival. Natural habitat loss and traffic activities are the main factors increasing its risk of extinction (Juniper and Parr 1998). In the JBMB, we directly observed and heard the species only once, in a wetland near the Vargem Limpa River in August 2016, outside the point counts $\left(22^{\circ} 20^{\prime} 39^{\prime \prime} \mathrm{S}, 049^{\circ} 00^{\prime} 54^{\prime \prime} \mathrm{W}\right)$.

Amazona aestiva (Linnaeus, 1758). Nationally and regionally Near Threatened. This species inhabits Cerrado, Chacos, and subtropical forests, and is restricted to primary forests with sufficiently tall trees for nesting (Collar et al. 2018b). It has been affected by illegal capture and traffic since the 1980s (BirdLife International 2018) and has suffered population decline due to fragmentation and deforestation (Collar et al. 2018b). It was recorded next to the JBMB main trail, at point no. 02 .

Rhynchotus rufescens (Temminck, 1815). Regionally Endangered. The species inhabits areas up to $2500 \mathrm{~m}$ above sea level. In tropical lowlands, it shows preference for wet grasslands and Savanna woodland. It is primarily an insectivore, but it also feeds on seeds, fruits, roots, and bulbs when insects are scarce. $R$. rufescens is considered sedentary and is subject to poaching for its meat. Populations in Brazil are in steady decline due to hunting pressures associated with habitat loss and poisoning by insecticides (Cabot et al. 2019). In the JBMB, we found this species in open grasslands in the regenerating area, especially at points no. 20-23.

Penelope superciliaris (Temminck, 1815). Regionally Near Threatened. P. superciliaris occupies a wide range of vegetal physiognomies, from Cerrado to dense and humid forests. It is a large generalist frugivore that forages in flocks of up to 10 individuals, maintaining a sedentary habit. It exhibits no major movement patterns throughout its life (del Hoyo and Kirwan 2018). In the JBMB, the species is abundant in Semideciduous Forest and was recorded at points no. 02-04.

Heliornis fulica (Boddaert, 1783). Regionally Endangered. This species lives in dense vegetation on the margins of water bodies (Bertram and Kirwan 2018), and the loss of riparian habitats is the main threat to its survival (Bressan 2009). There are few records of this species in São Paulo state, which makes its presence in the JBMB extremely valuable. We found one individual in a lake located on the western border of the JBMB, in its limit with the Municipal Zoological Garden of Bauru $\left(22^{\circ} 20^{\prime} 31^{\prime \prime} \mathrm{S}, 049^{\circ} 01^{\prime} 02^{\prime \prime} \mathrm{W}\right)$.

Crotophaga major Gmelin, 1788. Regionally Near Threatened. This species is relatively demanding regarding habitat quality, with few records existing in the state, because it is commonly found in aquatic macrophytes and hanging vegetation above water (Payne and de Juana 2018). The loss of most Riparian Forests due to construction of hydroelectric power plants in São Paulo state led to a significant decrease in its populations. This species was recorded in the JBMB surveys, in which 2 researchers reported the presence of 3 individuals in an area adjacent to Semideciduous Flooded Forest in November 2016 $\left(22^{\circ} 20^{\prime} 40^{\prime \prime} \mathrm{S}, 049^{\circ} 01^{\prime} 04^{\prime \prime} \mathrm{W}\right)$.

Bubo virginianus (Gmelin, 1788). Regionally Endangered. B. virgianius occupies forested habitats, such as Semideciduous Forests, secondary forests, and urban city parks. B. virginianus usually forages in open areas and feeds on insects, fishes, amphibians, birds, and small mammals. It is a resident species, although it includes some outlier subspecies populations migrating from North America when resources are scarce (Holt et al. 2018). Although it is not threatened at a global scale, it is considered Endangered in São Paulo due to population decline (São Paulo 2018). We recorded this species over several months, both in Forest Savanna and Semideciduous Forest, at points no. 03 and 12.

Herpsilochmus longirostris Pelzeln, 1868. Regionally Near Threatened and a Cerrado endemic. See the previous section on endemic species.

Neopelma pallescens (Lafresnaye, 1853). Regionally Near Threatened. This species is common in dense and tangled vegetation (Snow 2018), especially in Semideciduous and Gallery Forests. It inhabits the midstory stratum of Forest Savanna (1-5 m from the ground) and feeds mainly on insects and fruits (Willis and Oniki 2003). Because the species has narrowly defined habitat preferences, the main threats to it are related to fragmentation and habitat loss in São Paulo state. Therefore, $N$. pallescens is considered Endangered on the São Paulo Red List (São Paulo 2018). We recorded this species over 2 consecutive months (August and September 2017) in Forest Savanna remnants at points no. 08 and 09.

Discrimination between congeneric taxa. We recorded some congeneric species that are difficult to differentiate during surveys. Here, we list the diagnostic features that allowed these species to be distinguished, based on Grantsau (2010). 
Crypturellus obsoletus, Crypturellus parvirostris, and Crypturellus tataupa. We identified these species based on song. C. tataupa has a vocalization with trilled notes descending in frequency, which tends to be shorter than the songs of the other two species. C. parvirostris has long songs with whistled notes that are repeated in acceleration, ending in a decline in frequency and with repeated trilled notes at the end of the vocalization. $C$. obsoletus produces vocalizations with structure similar to $C$. parvirostris, but the final portion of its vocalization ends abruptly, with no repetition of notes. C. obsoletus was recorded at point no. 02, C. parvirostris was recorded at point no. 12 , and $C$. tataupa was recorded at point no. 16 .

Laterallus viridis and Laterallus melanophaius. Both species were identified by their songs. While L. melanophaius has a fast-trilling vocalization, $L$. viridis songs have introductory notes, with a slower pace of elements. L. melanophaius was found at point no. 15 , while L. viridis was found at point no. 01.

Leptotila verreauxi and Leptotila rufaxilla. L. verreauxi has a crown and neck with a greenish glow, olive-brown upper part, and a pale gray underpart that becomes white on the belly, and vocalizations with 2 low-frequency notes. In contrast, L. rufaxilla has a bluish gray crown, nape, and hindneck with a grayish purple glow, dark-brown upper part, and a greyish pink under part that becomes white on the belly, and low-pitched vocalizations with just 1 descending note. Both species was found at points no. 02-07.

Crotophaga major and Crotophaga ani. While C. ani has a uniform black color from the bill to the tail, $C$. major has dark-blue underparts and upper parts with a greenish sheen, prominent culmen, and white iris. We found $C$. major in a flooded field $\left(22^{\circ} 20^{\prime} 40^{\prime \prime} \mathrm{S}, 049^{\circ} 01^{\prime} 04^{\prime \prime}\right.$ $\mathrm{W})$ and $C$. ani in the open area of Savanna in regeneration, at points $17-24$.

Herpsilochmus atricapillus and Herpsilochmus longirostris. We found each species in distinct habitats. $H$. atricapillus was only present in Forest Savanna, while $H$. longirostris was only present in a fragment of Semideciduous Flooded Forest. Female individuals of $\mathrm{H}$. atricapillus have a black crown streaked with white dots, and a buff tinge on the breast, flank, and crissum. Meanwhile, females of $H$. longirostris have a bright orange head and neck, and bright-cinnamon underparts. We found $H$. atricapillus at points no. 03 and 07 , while $H$. longirostris was found at points no. 25-28.

Todirostrum poliocephalum and Todirostrum cinereum. Both species are distinguished by the bright yellow supraloral mark, which is present in T. poliocephalum and absent in T. cinereum. Furthermore, songs of T. poliocephalum have slower trill rates than those of $T$. cinereum. We found T. poliocephalum at point no. 03 and T. cinereum at points no. 03-07.
Elaenia flavogaster, Elaenia mesoleuca, and Elaenia obscura. These species have similar morphology. E. obscura was only found in a forest area, while E. flavogaster and E. mesoleuca were found in a regeneration area. Morphologically, E. mesoleuca and E. flavogaster are quite similar, differing in the crest (present only in $E$. flavogaster) and in the color of their underparts (greyish olive breast and pale yellow belly in E. flavogaster, and dull olive breast and greyish white belly in E. mesoleuca). In contrast, E. obscura is noticeably bigger than the other species, and has a remarkably short bill and darker upper parts than the other species. We recorded E. flavogaster at points no. 13-24, E. mesoleuca at points no. 23-24. and E. obscura at point no. 09.

Myiarchus ferox and Myiarchus swainsoni. Like the Elaenia spp., Myiarchus spp. have similar external morphology. Therefore, these congeneric species were distinguished based on their vocalizations. M. ferox has trilled elements in its song, while M. swainsoni produces short and frequency-modulated notes that sounds like a "hiccup." We recorded M. ferox at the edges of Semideciduous Forest (points no. 01 and 02), in the Forest Savanna (points 05-10), and in the regenerating area (points 17-24). We recorded M. swainsoni in the last points of the regenerating area (points 20-24).

Euphonia chlorotica and Euphonia violacea. These species are distinguished by the color of their throats. $E$. chlorotica has a dark-metallic blue throat, while $E$. violacea has a golden yellow throat. E. chlorotica was found at points no. 02-10, while E. violacea was only found at point no. 05 .

\section{Discussion}

Our results demonstrate that JBMB have a high species richness, which might be related to its location in the transitional area between the Atlantic Forest and Cerrado domains. We identified 9 species that are subject to some degree of threat and 11 endemic species ( 7 from Atlantic Forest and 4 from Cerrado). In addition, 31 species exhibited some degree of migratory behavior.

When comparing our results to other surveys in Semideciduous Forests, it is evident that the richness of the JBMB is greater than that in the more extensive areas of the interior Atlantic Forests of São Paulo. Donatelli et al. (2007) reported the presence of 65 and 64 species in fragments of 350 and 480 ha of Semideciduous Forest, respectively. We must take into account that their sampling effort was smaller than ours, as they spent 80 $\mathrm{h}$ using point counts in secondary-growth forests. However, our study documented higher richness, even when compared to other studies with longer sampling efforts, such as Aleixo and Vielliard (1995), who recorded 97 species in a 251 ha remnant over $235 \mathrm{~h}$ using point counts. The elevated richness (220 bird species) in our study could be due to the diverse habitats of the JBMB, which encompasses Semideciduous Forests and other 
Atlantic forest phytophysiognomies. Moreover, the high degree of anthropogenic activities in the surrounding areas might also enhance species richness by the inclusion of some resilient taxa to disturbed environments, especially in tropical environments (Aleixo 1999, Mulwa et al. 2012, Asefa et al. 2017).

Few studies have been conducted in Forest Savanna and have recorded much lower richness, with fewer than 100 species being associated exclusively with this physiognomy (Caverzere et al. 2011). More species were detected in studies that included other Savanna physiognomies, such as grasslands and shrublands. Manica et al. (2010) recorded 160 bird species in 472 ha Cerrado fragment over $95 \mathrm{~h}$ using qualitative surveys. However, of all of these species, only 28 were found in Forest Savanna physiognomy. Other studies encompassing more extensive areas over longer sampling periods obtained a similar number of species to our study. For instance, Motta-Júnior et al. (2008) recorded 231 species over 580 $\mathrm{h}$ of sampling in 2,300 ha of Cerrado grasslands using point counts. Lucindo et al. (2015) recorded 226 species in $380 \mathrm{~h}$ of sampling 2,712 ha mixed physiognomies of Cerrado using transect counts.

Of the non-passerines, the greatest number of species were represented by the families Trochilidae, Accipitridae, and Columbidae. Their presence is associated with the fragmented areas and their conspicuous behavior makes them easy to be detected during sampling (Sick 1997). Columbidae are predominantly granivores, while Accipitridae are carnivores. Such feeding behaviors are favored in open habitats, such as Forest Savanna and the regenerating areas of the JBMB (Chettri et al. 2004, Winkler et al. 2015). The richness of the Trochilidae is indicative of the abundance of floral resources, because species of this family are predominantly nectarivores (Wilman et al. 2014). The presence of several physiognomies in the study area might provide diverse flowering plants at different times of the year, especially in the transition periods between dry and rainy seasons (Pirani et al. 2009). Finally, the presence of a network of wetlands, lakes, and rivers in the study area favored the presence of species dependent on aquatic environments, such as those of the families Ardeidae and Rallidae (7 species each).

Among Passeriformes, Tyrannidae and Thraupidae had more species than the other familes.greatest number of species were represented by the families Both are typical of Neotropical bird communities, and the family Tyrannidae is one of the largest groups in the region (Ridgely and Tudor 1994, Sick 1997). This phenomenon might be associated with the generalist habit of several species in this family (Sick 1997), which allows them to access several types of habitats. On the other hand, several species of Thraupidae feed on fruits and act as seed dispersers (Ridgely and Tudor 1989) and are important for maintaining plant diversity. The richness of Thraupidae can also be associated with recent taxonomic changes proposed by Piacentini et al. (2015) which subsumed the formerly separate Emberizidae and Coerebidae as subfamilies within Thraupidae. This fusion increased the number of species of Thraupidae from 99 species to 173 species. Nonetheless, the richness of frugivorous and granivorous birds could indicate the variety of the habitats available in the region.

When comparing our survey to a previous survey on Forest Savanna in the JBMB area, we found 28 species that were not reported by Caverzere et al. (2011). Of these, we recorded 8 endemic species (5 to Atlantic Forest and 3 for Cerrado), and 2 threatened taxa, which are noteworthy records. However, these authors reported 10 species that we did not find during our study. Yet, we observed 8 of these species in other environments near the JBMB. The 2 other species unique to the survey by Caverzere et al. (2011) were Leptodon cayanensis and Hylophilus poicilotis. The absence of records on $L$. cayanensis in our survey might be related to its tends to occupy a large territory (Brown and Amadon 1989). Its movement across the landscape might make it difficult to record this species in the study area. In contrast, the record of $H$. poicilotis seems to be questionable, since we recorded only Hylophilus amaurocephalus, a very similar congeneric species. H. amaurocephalus was also recorded by Rangel de Almeida et al. (2010) at the Bauru Ecological Station, with this being the only record of the genus in the vicinity of our survey site. In addition, citizen science databases also show records of only $H$. amaurocephalus in Bauru (Wikiaves 2019). The nearest record of $H$. poicilotis is from the city of Campinas, located more than $200 \mathrm{~km}$ away from the JBMB (eBird 2019). In comparison, H. amaurocephalus is quite common in the meso-region of Bauru, with records in the nearby cities (eBird 2019). Therefore, we suggest that the record of $H$. poicilotis in the JBMB is incorrect, because the songs of these 2 species are similar. Because H. poicilotis is endemic to the Atlantic Forest (Bencke et al. 2006), it is not likely to inhabit an area of Forest Savanna, even in a transitional area such as the JBMB.

According to Somenzari et al. (2018), Brazil has 198 migratory species, which corresponds to approximately $10 \%$ of all species found in the country. In our surveys, we observed 31 species (14.1\%) exhibiting migratory behavior. For species defined as migrants, the records of Chordeiles minor and Chaetura meridionalis are of note. C. minor reproduces in North and Central America, migrates to South America in September, and returns to its locations of origin in April (Cleere 2018). C. meridionalis reproduces in Brazil during the austral summer and migrates north to Panama, Venezuela, and French Guiana in the winter (del Hoyo et al, 2018).

Records of endemic species is important because they are negatively affected by habitat fragmentation and loss (Aleixo and Vielliard 1995, Anjos 2001). These species can be considered habitat specialists, and their presence in our survey can be an indicative of the quality of forest remnants. In the Semideciduous Forest, we recorded 7 Atlantic Forest endemics. Few endemic species were 
recorded in our survey compared to other surveys: 33 spp. by Faria et al. (2006), 43 spp. by Ubaid (2009), and 22 spp. by Vianna et al. (2017). Antunes (2007) recorded the disappearance of 9 endemic species over 30 years in a fragment of Semideciduous Forest, while the abundance of another 9 species significantly declined. Alternatively, we recorded 4 Cerrado endemic species, which was more than that recorded by Cavarzere et al. (2011) at the same location; Cavarzere et al. recorded only Antilophia galeata as endemic to the Cerrado. Both Cyanocorax cristatellus and Saltatricula atricollis are Cerrado endemic species that we found exclusively in the regenerating area, which is because they normally inhabit open physiognomies of savanna. Cavarzere et al. (2011) did not found these species because they restricted their survey to closed physiognomies (forest savanna and semideciduous forest). Our study obtained a similar richness of endemic Cerrado taxa when compared to previous studies: 6 spp. by Motta-Júnior et al. (2008), 6 spp. by Manica et al. (2010), and 6 spp. by Lucindo et al. (2015). The presence of such endemic taxa could point to the capacity of the environment to provide sufficient resources across seasons (Antunes 2007) and might be related to the different stages of vegetation regeneration.

The richness of birds recorded in the JBMB might be related to the large area of continuous native forest (803 ha) and the presence of areas at different stages of forest regeneration. The number of species in a given location is associated with habitat heterogeneity, which is an important predictor of bird richness (Stein et al. 2014, Stirnemann et al. 2015) and is important for local community maintenance (Develey et al. 2010). A greater diversity of habitats is related to a greater diversity of resources, which supports a greater number of species. Thus, the regenerating area of the JBMB supports species exclusive to open areas, which dominate such places. Meanwhile, the savanna remnants surrounding the regenerating area could also contribute to increase the number of the species in this location, which could improve the regional richness by landscape supplementation (Dunning et al. 1992). Birds dwelling in closed environments could use the regeneration area to gather supplementary resources in adjacent boundaries (Tubelis et al. 2004, Brotons et al. 2005), which could explain the presence of forest-dwelling species in an open area.

In conclusion, the JBMB is an important area with a richness of bird species higher than other surrounding conservation units. Such richness is attributed to the transition between the Atlantic Forest and Cerrado, as well as to the variety of physiognomies and successional stages in the area favoring the distribution of several taxa within a restricted area. Although we only recorded few endemic and threatened species, these records demonstrate the capacity of the local environment to support species that require specific ecological resources. Our findings also contribute to the knowledge of avian richness in transitional areas, demonstrating the importance of this type of environment for the maintenance of bird diversity.

\section{Acknowledgements}

We thank Luiz Carlos de Almeida Neto, director of JBMB, for providing collaboration and logistical support. We also thank the public servants of JBMB for providing help during the surveys. We acknowledge the language editing support provided by Editage, which reviewed the grammar of this manuscript.

\section{Author Contributions}

GSC, RMV, and RWG contributed equally with the study design, data collection, discussion, and writing of the manuscript. RJD contributed with the supervision of this project, and with the correction of the final manuscript.

\section{References}

Aleixo A (1999) Effects of selective logging on a bird community in the Brazilian Atlantic Forest. Revista Brasileira de Zoologia 12 (3): 493-511. https://doi.org/10.2307/1370183

Aleixo A, Vielliard JME (1995) Composição e dinâmica da avifauna da mata de Santa Genebra, Campinas, São Paulo, Brasil. Revista Brasileira de Zoologia 12 (3): 493-511. https://dx.doi.org/10.1590/ S0101-81751995000300004

Alvares CA, Stape JL, Sentelhas PC, Gonçalves JLM, Sparovek G (2013) Köppen's climate classification map for Brazil. Meteorologische Zeitschrift 22 (6): 711-728. https://doi.org/10.1127/09412948/2013/0507

Anjos L (2001) Bird communities in five Atlantic Forest fragments in southern Brazil. Ornitologia Neotropical 12 (1): 11-27.

Anjos L (2018) Curl-crested Jay (Cyanocorax cristatellus). Handbook of the Birds of the World Alive. Lynx Edicions, Barcelona. https:// www.hbw.com/node/60715. Accessed on: 2018-1-29.

Antunes AZ (2007) Riqueza e dinâmica de aves endêmicas da Mata Atlântica em um fragmento de floresta estacional semidecidual no sudeste do Brasil. Revista Brasileira de Ornitologia 15 (1): 61-68.

Asefa A, Davies AB, McKechnie AE, Kinahan AA, van Rensburg BJ (2017) Effects of anthropogenic disturbance on bird diversity in Ethiopian Montane forests. The Condor 119 (3): 416-430. https:// doi.org/10.1650/CONDOR-16-81.1

Baker J, French K, Whelan RJ (2002) The edge effect and ecotonal species: bird communities across a natural edge in southeastern Australia. Ecology 83 (11): 3048-3059. https://doi. org/10.1890/0012-9658(2002)083[3048:TEEAES]2.0.CO;2

Bauru (2000) Lei N $\mathrm{N}^{\circ}$ 4605, de 27 de novembro de 2000. Denomina e regulamenta os usos na Área de Proteção Ambiental Municipal Vargem Limpa - Campo Novo. http://www2.bauru.sp.gov.br/ arquivos/sist_juridico/documentos/leis/lei4605.pdf. Accessed on: 2018-2-21.

Bencke GA, Mauricio GN, Develey PF, Goerck JM (2006) Áreas importantes para a conservação das aves no Brasil: parte 1-estados do domínio da Mata Atlântica. SAVE Brasil, São Paulo, 494 pp.

Bertram BCR, Kirwan GM (2018) Sungrebe (Heliornis fulica). Handbook of the Birds of the World Alive. Lynx Edicions, Barcelona. https://www.hbw.com/node/53707. Accessed on: 2018-1-28.

Blondel J, Ferry C, Frochot B (1981) Point counts with unlimited distance. Studies in Avian Biology 6: 414-420.

Bressan PM, Kierulff MCM, Sugieda AM (2009) Fauna ameaçada de extinção no Estado de São Paulo. Fundação Parque Zoológico de São Paulo, Secretaria do Meio Ambiente, São Paulo. http://www. ib.usp.br/ 1fsilveira/pdf/1_2010_faunaextsp.pdf. Accessed on: 2018-3-15.

Brewer D (2018) Black-throated Saltator (Saltator atricollis). Hand- 
book of the Birds of the World Alive. Lynx Edicions, Barcelona. https://www.hbw.com/node/62206. Accessed on: 2018-1-29.

Brewer D (2018) Red-eyed Vireo (Vireo olivaceus). Handbook of the Birds of the World Alive. Lynx Edicions, Barcelona. https://www. hbw.com/node/61266. Accessed on: 2018-2-26.

Brotons L, Wolff A, Paulus G, Martin JL (2005) Effect of adjacent agricultural habitat on the distribution of passerines in natural grasslands. Biological Conservation 124 (3): 407-414. https://doi. org/10.1016/j.biocon.2005.01.046

Brown L, Amadon D (1989) Eagles, Hawks and Falcons of the World. Wellfleet, New York, 946 pp.

Cabot J, Christie DA, Jutglar F, Sharpe CJ, Garcia EFJ (2019) Redwinged Tinamou (Rhynchotus rufescens). Handbook of the Birds of the World Alive. Lynx Edicions, Barcelona. https://www.hbw. com/node/52438. Accessed on: 2019-3-28.

Cavarzere V, Moraes GP, Dalbeto AC, de Maciel FG, Donatelli RJ (2011) The birds of the cerradão woodland, an overlooked forest of the Cerrado region. Papéis Avulsos de Zoologia 51 (17): 259-273. http://dx.doi.org/10.1590/S0031-10492011001700001

Cavassan O (2013) Bauru: terra de cerrado ou floresta? Ciência Geográfica 17 (17): 46-54.

Chettri N, Deb DC, Sharma E, Jackson R (2005) The relationship between bird communities and habitat. Mountain Research and Development 25 (3): 235-243. https://doi.org/10.1659/02764741(2005)025[0235:TRBBCA]2.0.CO;2

Cleere N (2018) Common Nighthawk (Chordeiles minor). Handbook of the Birds of the World Alive. Lynx Edicions, Barcelona. https:// www.hbw.com/node/55166. Accessed on: 2018-2-27.

Collar N, Boesman P, Sharpe CJ (2018a) Blue-winged Macaw (Primolius maracana). Handbook of the Birds of the World Alive. Lynx Edicions, Barcelona. https://www.hbw.com/node/54626. Accessed on: 2018-1-29.

Collar N, Kirwan GM, Boesman P (2018b) Turquoise-fronted Amazon (Amazona aestiva). Handbook of the Birds of the World Alive. Lynx Edicions, Barcelona. https://www.hbw.com/node/54749. Accessed on: 2018-2-26.

Costa FJV, Monteiro KRG (2016) Guia de identificação das aves traficadas no Brasil. BECONN, Florianópolis, 200 pp.

Craveiro RB, Miyaki CY (2000) Analysis of the genetic variability of Propyrrhura maracana (Psittaciformes, Aves) using DNA fingerprinting. Ararajuba 8 (2): 79-84.

Dangerfield JM, Pik AJ, Britton D, Holmes A, Gillings M, Oliver I, Briscoe D, Beattie AJ (2003) Patterns of invertebrate biodiversity across a natural edge. Austral Ecology 28 (3): 227-236. https:// doi.org/10.1046/j.1442-9993.2003.01240.x

Dário FR, Vincenzo MCV, Almeida AF (2002) Avifauna em fragmentos da Mata Atlântica. Ciência Rural 32 (6): 989-996. https://doi. org/10.1590/S0103-84782002000600012

del Hoyo J, Collar N (2018) Eastern Slaty Thrush (Turdus subalaris). Handbook of the Birds of the World Alive. Lynx Edicions, Barcelona. https://www.hbw.com/node/1344026. Accessed on: 2018$1-29$.

del Hoyo J, Collar N, Kirwan GM (2018) Southern Swift (Chaetura meridionalis). Handbook of the Birds of the World Alive. Lynx Edicions, Barcelona. https://www.hbw.com/node/467189. Accessed on: 2018-2-27.

del Hoyo J, Kirwan GM (2018) Rusty-margined Guan (Penelope superciliaris). Handbook of the Birds of the World Alive. Lynx Edicions, Barcelona. https://www.hbw.com/node/53285. Accessed on: 2018-2-26.

Develey PF, Pongiluppi T (2010) Impactos potenciais na avifauna decorrentes das alterações propostas para o Código Florestal Brasileiro. Biota Neotropica 10 (4): 43-46. https://doi.org/10.1590/ S1676-06032010000400005

Donatelli RJ, Ferreira CD, Dalbeto AC, Posso SR (2007) Análise comparativa da assembleia de aves em dois remanescentes florestais no interior do Estado de São Paulo, Brasil. Revista Brasileira de Zoologia 24 (2): 362-375. https://doi.org/10.1590/
S0101-81752007000200015

Dunning JB, Danielson BJ, Pulliam HR (1992) Ecological processes that affect populations in complex landscapes. Oikos 65 (1): 169-175. https://doi.org/10.2307/3544901

Durigan G, Baitello JB, Franco GADC, Siqueira MF (2004) A vegetação dos remanescentes de cerrado no Estado de São Paulo. In: Bittencourt MD, Mendonça RR (Eds) Viabilidade de conservação dos remanescentes de Cerrado no Estado de São Paulo. Annablume, São Paulo, 29-56.

eBird (2019) Verdinho-coroado Hylophilus poicilotis. Available in: https://ebird.org/species/rucgre1. Accessed on: 2019-3-20.

Faria CMA, Rodrigues M, Amaral FQ, Módena E, Fernandes AM (2006) Aves de um fragmento de Mata Atlântica no Alto Rio Doce, Minas Gerais: colonização e extinção. Revista Brasileira de Zoologia 23 (4): 1217-1230. http://dx.doi.org/10.1590/S010181752006000400032

Grantsau RKH (2010) Guia completo para identificação das aves do Brasil. Vento Verde, São Carlos, 624 pp.

Hilty S (2018) Black-faced Tanager (Schistochlamys melanopis). Handbook of the Birds of the World Alive. Lynx Edicions, Barcelona. https://www.hbw.com/node/61572. Accessed on: 2018-1-29.

Holt DW, Berkley R, Deppe C, Enríquez Rocha P, Petersen JL, Rangel Salazar JL, Segars KP, Wood KL, Marks JS (2018) Great Horned Owl (Bubo virginianus). Handbook of the Birds of the World Alive. Lynx Edicions, Barcelona. https://www.hbw.com/ node/55006. Accessed on: 2018-2-26.

ICMBIO/MMA (2018) Livro vermelho da fauna ameaçada de extinção: Volume 1. Instituto Chico Mendes de Conservação da Biodiversidade, Brasília, 492 pp.

IUCN (2019). The IUCN Red List of Threatened Species. https:// www.iucnredlist.org/ Accessed on: 2018-1-22

Juniper T, Parr M (1998) Parrots: a Guide to the Parrots of the World. Christopher Helm Publisher, London, 584 pp.

Kark S, Van Rensburg BJ (2006) Ecotones: marginal or central areas of transition? Israel Journal of Ecology \& Evolution 52 (1): 29-53. https://doi.org/10.1560/IJEE.52.1.29

Kark S, Allnutt TF, Levin N, Manne LL, Williams PH (2007) The role of transitional areas as avian biodiversity centres. Global Ecology and Biogeography 16 (2): 187-196. https://doi.org/10.1111/j.14668238.2006.00274.x

Kronka FJN, Nalon MA, Matsukuma CK, Pavão M, Guillaumon JR, Cavalli AC, Gianotti E, Ywane MSSI, Lima LMPR, Montes J, Cali IHD, Haack PG (1998) Áreas de domínio do Cerrado no estado de São Paulo. Instituto Florestal, São Paulo, 84 pp.

Kucherova SV, Mirkin BM (2001) On methods for analyzing ecotones of forest margins. Russian Journal of Ecology 32 (5): 310-314. https://doi.org/10.1023/A:1011918025846

Kunin WE (1998) Biodiversity at the edge: a test of the importance of spatial "mass effects" in the Rothamsted Park Grass experiments. Proceedings of the National Academy of Sciences of the United States of America 95 (1): 207-212. https://doi.org/10.1073/ pnas.95.1.207

Lloyd KM, McQueen AAM, Lee BJ, Wilson RCB, Walker S, Wilson JB (2000) Evidence on ecotone concepts from switch, environmental and anthropogenic ecotones. Journal of Vegetation Science 11 (6): 903-910. https://doi.org/10.2307/3236560

Lucindo AS, Antunes AZ, Kanashiro MM, Dias MM (2015) Birds at Santa Bárbara Ecological Station, one of the last Cerrado remnants in the state of São Paulo, Brazil. Biota Neotropica 15 (4): e0155. http://doi.org/10.1590/1676-06032015015514

Manica LT, Telles M, Dias MM (2010) Bird richness and composition in a Cerrado fragment in the State of São Paulo. Brazilian Journal of Biology 70 (2): 243-254. https://doi.org/10.1590/S151969842010005000001

Moritz C, Patton JL, Schneider CJ, Smith AR (2000) Diversification of rainforest faunas: an integrated molecular approach. Annual Review of Ecology and Systematics 31: 533-563. https://oi.org/ 10.1146/annurev.ecolsys.31.1.533 
Motta-Junior JC, Granzinolli MAM, Develey PF (2008) Aves da estação ecológica de Itirapina, estado de São Paulo, Brasil. Biota Neotropica 8 (3). http://doi.org/10.1590/S1676-06032008000300019

Mulwa RK, Böhning-Gaese K, Schleuning M (2012) High bird species diversity in structurally heterogeneous farmland in western Kenya. Biotropica 44 (6): 801-809. https://doi.org/10.1111/j.17447429.2012.00877.x

Myers N, Mittermeier RA, Mittermeier CG, Fonseca GAB, Kent J (2000) Biodiversity hotspots for conservation priorities. Nature 403 (6772): 853-858. https://doi.org/10.1038/35002501

Nunes MFC, Galetti M (2007) Use of forest fragments by bluewinged macaws (Primolius maracana) within a fragmented landscape. Biodiversity and Conservation 16 (4): 953-967. https://doi. org/10.1007/s10531-006-9034-9

Odum EP (1953) Fundamentals of ecology. W.B. Saunders Company, Philadelphia, $383 \mathrm{pp}$

Pacheco JF (2003) As aves da Caatinga: uma análise histórica do conhecimento. In: Silva JMC, Tabarelli M, Fonseca MT, Lins LV (Eds) Biodiversidade da Caatinga: áreas e ações prioritárias para a conservação. Ministério do Meio Ambiente, Brasília, 189-250.

Payne R, de Juana E (2018) Greater Ani (Crotophaga major). Handbook of the Birds of the World Alive. Lynx Edicions, Barcelona. https://www.hbw.com/node/54906. Accessed on: 2018-1-29.

Piacentini VDQ, Aleixo A, Agne CE, Maurício GN, Pacheco JF, Bravo GA, Brito GRR, Naka LN, Olmos F, Posso S, Silveira LF, Betini GS, Carrano E, Franz I, Lees AC, Lima LM, Pioli D, Schunck F, Amaral FR, Bencke GA, Cohn-Haft M, Figueiredo LFA, Straube FC, Cesari E (2015) Annotated checklist of the birds of Brazil by the Brazilian Ornithological Records Committee. Revista Brasileira de Ornitologia 23 (2): 90-298.

Pirani FR, Sanchez M, Pedroni F (2009) Fenologia de uma comunidade arbórea em cerrado sentido restrito, Barra do Garças, MT, Brasil. Acta Botanica Brasilica 23 (4): 1096-1109. http://doi.org/ 10.1590/S0102-33062009000400019

Rangel de Almeida EM, Toniato MTZ, Durigan G (2010) Estação Ecológica de Bauru: plano de manejo. Fundação Florestal da Secretaria do Meio Ambiente do Estado de São Paulo, São Paulo. 200 pp.

Remsen Jr JV, Kirwan GM (2018) White-eyed Foliage-gleaner (Automolus leucophthalmus). Handbook of the Birds of the World Alive, Lynx Edicions. Barcelona. https:/www.hbw.com/node/ 56573. Accessed on: 2018-1-29.

Ridgely RS, Tudor G (1989) The Birds of South America: the oscine passerines. University of Texas Press, Austin, 516 pp.

Ridgely RS, Tudor G (1994) The Birds of South America: the suboscine passerines. Texas, University of Texas Press, Austin, $814 \mathrm{pp}$.

Rodrigues RR (2000) Florestas ciliares: uma discussão nomenclatural das formações ciliares. In: Rodrigues RR, Leitão Filho HF (Eds) Matas ciliares: conservação e recuperação. Editora da Universidade de São Paulo, São Paulo, 91-99.

Santos AJ (2003) Estimativas de riqueza em espécies. In: Cullen Jr L, Rudran R, Valladares-Padua C (Eds) Métodos de estudos em biologia da conservação e manejo da vida silvestre. Editora da Universidade Federal do Paraná, Brasil, 19-41.

São Paulo (2017) Resolução SMA N 146, de 08 de novembro de 2017. Institui o Mapa de Biomas do Estado de São Paulo, e dá outras providências. http://arquivo.ambiente.sp.gov.br/legislacao/2017/11/ resolucao-sma-146-2017.pdf. Accessed on: 2018-2-22.

São Paulo (2018) Decreto $N^{\circ}$ 63.853, de 27 de novembro de 2018. Declara as espécies da fauna silvestre no Estado de São Paulo regionalmente extintas, as ameaçadas de extinção, as quase ameaçadas e as com dados insuficientes para avaliação, e dá providências correlatas. http://arquivos.ambiente.sp.gov.br/fauna/2016/12/ Decreto.Estadual.60133.14.fauna_.ameaçada.pdf. Accessed on: 2018-2-21.

Schilthuizen M (2000) Ecotone: speciation prone. Trends in Ecology \& Evolution 15 (4): 130-131. https://doi.org/10.1016/S0169-5347 (00)01839-5
Sick H (1997) Ornitologia brasileira. Nova Fronteira, Rio de Janeiro, $862 \mathrm{pp}$.

Silva JMC, Bates JM (2002) Biogeographic patterns and conservation in the South American Cerrado: a tropical savanna hotspot. Bioscience 52 (3): 225-233. https://doi.org/10.1641/0006-3568(2002) 052[0225:BPACIT]2.0.CO;2

Snow D (2018) Pale-bellied Tyrant-manakin (Neopelma pallescens) Handbook of the Birds of the World Alive. Lynx Edicions, Barcelona. https://www.hbw.com/node/57108. Accessed on: 2018-2-20.

Snow D, de Juana E (2018) Helmeted Manakin (Antilophia galeata). Handbook of the Birds of the World Alive. Lynx Edicions, Barcelona. https://www.hbw.com/node/57079. Accessed on: 2018-2-26.

Snow D, Kirwan GM (2018) Rufous-capped Motmot (Baryphthengus ruficapillus). Handbook of the Birds of the World Alive. Lynx Edicions, Barcelona. https://www.hbw.com/node/55824. Accessed on: 2018-1-29.

Somenzari M, Amaral PP, Cueto VR, Guaraldo AC, Jahn AE, Lima DM, Lima PC, Lugarini C, Machado CG, Martinez J, Nascimento JLX, Pacheco JF, Paludo D, Prestes NP, Serafini PP, Silveira LF, Sousa AEBA, Sousa NA, Souza MA, Telino-Júnior WR, Whitney BM (2018) An overview of migratory birds in Brazil. Papéis Avulsos de Zoologia 58: e20185803. https://doi.org/ 10.11606/ $1807-0205 / 2018.58 .03$

Stein A, Gerstner K, Kreft H (2014) Environmental heterogeneity as a universal driver of species richness across taxa, biomes and spatial scales. Ecology Letters 17 (7): 866-880. https://doi.org/ 10.1111/ele.12277

Stirnemann IA, Ikin K, Gibbons P, Blanchard W, Lindenmayer DB (2015) Measuring habitat heterogeneity reveals new insights into bird community composition. Oecologia 177 (3): 733-746. https://doi.org/10.1007/s00442-014-3134-0

Tubelis DP, Cowling A, Donnelly C (2004) Landscape supplementation in adjacent savannas and its implications for the design of corridors for forest birds in the central Cerrado, Brazil. Biological Conservation 118 (3): 353-364. https://doi.org/10.1016/j. biocon.2003.09.014

Ubaid FK (2009) Dinâmica da avifauna em dois remanescentes florestais do interior do Estado de São Paulo, Brasil. MSc disertation. Universidade Estadual Paulista Júlio de Mesquita Filho, Botucatu, $144 \mathrm{pp}$.

Veloso HP (1992) Sistema fitogeográfico. In: Veloso HP, Oliveira-Filho LD, Vaz AMSF, Lima MPM, Marquete R, Brazao JEM (Eds) Manual técnico da vegetação brasileira. Fundação Instituto Brasileiro de Geografia e Estatística, Rio de Janeiro, 9-38.

Vianna RM, Donatelli RJ, Whitacker R, Martins RM, SementiliCardoso G (2017) Dynamics of the bird communities in two fragments of Atlantic Forest in São Paulo, Brazil. Biologia 72 (8): 913-926. https://doi.org/10.1515/biolog-2017-0101

Vielliard JEM, Silva WR (1990) Nova metodologia de levantamento quantitativo de avifauna e primeiros resultados no interior do Estado de São Paulo, Brasil. In: Mendes S (Ed) Anais do IV Encontro Nacional de Anilhadores de Aves, Recife, 117-151.

Walther B (2018) Yellow-lored Tody-lycatcher (Todirostrum poliocephalum). Handbook of the Birds of the World Alive. Lynx Edicions, Barcelona. https://www.hbw.com/node/57300. Accessed on: 2018-1-29.

Weiser VL (2007) Árvores, arbustos e trepadeiras do cerradão do Jardim Botânico Municipal de Bauru, SP. PhD dissertation, Instituto de Biologia, Campinas, 100 pp.

Weller AA, Kirwan GM, Boesman P (2018) Violet-capped Woodnymph (Thalurania glaucopis). Handbook of the Birds of the World Alive. Lynx Edicions, Barcelona. https://www.hbw.com/ node/55463. Accessed on: 2018-1-29.

Whitney B, de Juana E, Kirwan GM (2018) Rufous Gnateater (Conopophaga lineata). Handbook of the Birds of the World Alive. Lynx Edicions, Barcelona. https://www.hbw.com/ node/56925 Accessed on: 2018-1-29.

Wikiaves (2019) Espécies em Bauru/SP. https://www.wikiaves.com. 
br/especies .php? $\mathrm{t}=\mathrm{c} \& \mathrm{c}=3506003$. Accessed on: 2019-2-22.

Willis EO (2006) Protected cerrado fragments grow up and lose even metapopulational birds in central São Paulo, Brazil. Brazilian Journal of Biology 66 (3): 829-837. http://doi.org/10.1590/S151969842006000500008

Willis EO, Oniki Y (2003) Aves do Estado de São Paulo. Editora Divisa, Rio Claro, 398 pp.

Wilman H, Belmaker J, Simpson J, de la Rosa C, Rivadeneira MM, Jetz W (2014) EltonTraits 1.0: Species-level foraging attributes of the world's birds and mammals. Ecology 95 (7): 2027-2027. https://doi.org/10.1890/13-1917.1

Winkler DW, Billerman SM, Lovette IJ (2015) Bird Families of the World: an Invitation to the Spectacular Diversity of Birds. Lynx Edicions, Barcelona, $600 \mathrm{pp}$.

Zimmer K, Isler ML (2018) Large-billed Antwren (Herpsilochmus longirostris). Handbook of the Birds of the World Alive. Lynx Edicions, Barcelona. https://www.hbw.com/node/56757. Accessed on: 2018-2-26. 